\title{
Advances and New Applications Using the Acousto-Optic Effect in Optical Fibers
}

\author{
Alexandre A. P. POHL ${ }^{1 *}$, Roberson A. OLIVEIRA ${ }^{2}$, Ricardo E. DA SILVA ${ }^{1}$, \\ Carlos A. F. MARQUES ${ }^{3}$, Paulo de Tarso NEVES JR. ${ }^{1}$, Kevin COOK $^{4}$, \\ John CANNING ${ }^{4}$, and Rogério N. NOGUEIRA ${ }^{3}$
}

\author{
${ }^{1}$ Advanced Telecommunications Laboratory, Federal University of Technology - Paraná, Curitiba, Brazil \\ ${ }^{2}$ Volvo GTT, Advanced Technology \& Research, Curitiba, Brazil \\ ${ }^{3}$ Instituto de Telecomunicações, Pólo de Aveiro, 3810-193, Aveiro, Portugal \\ ${ }^{4}$ Interdisciplinary Photonics Labs, School of Chemistry, University of Sydney, New South Wales 2006, Australia \\ *Corresponding author: Alexandre A. P. POHL_ E-mail: pohl@utfpr.edu.br
}

\begin{abstract}
This work presents a short review of the current research on the acousto-optic mechanism applied to optical fibers. The role of the piezoelectric element and the acousto-optic modulator in the excitation of flexural and longitudinal acoustic modes in the frequency range up to $1.2 \mathrm{MHz}$ is highlighted. A combination of the finite elements and the transfer matrix methods is used to simulate the interaction of the waves with Bragg and long period gratings. Results show a very good agreement with experimental data. Recent applications such as the writing of gratings under the acoustic excitation and a novel viscometer sensor based on the acousto-optic mechanism are discussed.
\end{abstract}

Keywords: Acousto-optics, fiber gratings, optical fibers, optical sensors

Citation: Alexandre A. P. POHL, Roberson A. OLIVEIRA, Ricardo E. DA SILVA, Carlos A. F. MARQUES, Paulo de Tarso NEVES JR., Kevin COOK, et al., "Advances and New Applications Using the Acousto-Optic Effect in Optical Fibers," Photonic Sensors, vol. 3, no. 1, pp. 1-25, 2013.

\section{Introduction}

The acousto-optic effect has been successfully used since the early 1980's in the design and construction of a variety of optical fiber devices, such as frequency shifters, tapers, couplers, filters and modulators. When acoustic waves propagate in fibers, they cause strain along the guide and change the electrical permitivitty of the material by means of the mechanism known as photoelasticity [1]. For instance, the strain field causes alterations in the impermeability tensor, which is converted into changes in the effective refractive index $n_{\text {eff, }}$ described as

$$
\Delta\left(\frac{1}{n_{\mathrm{eff}}^{2}}\right)_{i}=\sum_{j=1}^{6} p_{i j} \varepsilon_{j}, \quad i=1,2,3, \cdots, 6
$$

where $p_{i j}$ are the components of the elasto-optic tensor, and $\varepsilon_{j}$ is the $j$-th component of the strain field. By controlling the frequency and amplitude of the propagating acoustic wave, one is able to modulate the characteristics of the light guided in the structure.

The study of the acousto-optic interaction in fibers began with the understanding of the propagation of acoustic waves in rod-like structures.

Received: 22 October 2012 / Revised version: 12 November 2012

(C) The Author(s) 2013. This article is published with open access at Springerlink.com 
The equations describing such propagation were first derived by Pochhammer in 1876 [2], and independently by Chree in 1889 [3] and are based on the solution of a boundary value problem in the theory of elasticity. Several textbooks dealing with the generation, processing and detection of acoustic signals are available, in which the propagation of acoustic waves in cylindrical structures is approached [4-6]. A detailed description of the acoustic modes propagating in a linear elastic isotropic cylindrical structure can also be found in Thurston [7], who studied the mechanical resonant modes in rod waveguides. His work presented the solutions and limitations of guiding in structures with different configurations such as waveguides without cladding, with thin cladding and infinite cladding. From these studies and references, one learns that acoustic modes propagating in guides can be largely classified as longitudinal, torsional and flexural waves. And for the propagation in uniform thin rod-like structures, such as optical fibers, the lowest order acoustic modes are of particular importance.

The basic device for generating acoustic waves in fibers makes use of an acousto-optic modulator, whose design and principle of operation were first proposed and patented by Zemon and Dakss in 1978 [8]. Since then, piezoelectric transducers $[9,10]$ have been widely utilized as drivers for the excitation of acoustic modes. In combination with glass or metal horns, which have the purpose of efficiently coupling the waves into the fibers, they form the basic building block of the acousto-optic modulator $[8,11]$. When the piezo material is driven by an electrical modulating signal, it induces acoustic waves in the horn and the fiber, modulating the intensity and spectral characteristics of the guided light by means of changes in the fiber refractive index and the light path.

Generally, acousto-optic applications utilize either flexural or longitudinal acoustic waves to control the properties of guided light, although some also use torsional waves [12, 13]. For instance, flexural waves produce periodic microbends in the fiber and cause energy transfer between optical modes. The efficiency of such coupling depends basically on the amplitude and frequency of the excitation produced by the transducer, which must provide the specific acoustic wavelength that corresponds to the beat length between the optical modes that take part in the coupling process [14]. The additional use of Bragg and long period gratings inscribed in fibers expand the range of application possibilities, allowing the modulation and switching of the grating reflection and transmission characteristics [15].

Though the acousto-optic interaction in fibers covers a large range of applications, this paper focuses mostly on the interaction of such waves with Bragg and long period fiber gratings with excitation in the frequency range from $50 \mathrm{kHz}$ to $1200 \mathrm{kHz}$, with a brief description, for the sake of completeness, of the optical mode coupling case, where no gratings are present in the fiber. The paper is divided as follows: Section 2 describes the main parameters of acoustic waves, their excitation technique using piezoelectric elements, the acoustic modes which are of interest for the acousto-optic interaction and the measurement of flexural and longitudinal modes; Section 3 provides the reader with a brief description of the acousto-optic interaction in fibers (without gratings) that leads to the coupling between optical modes; Section 4 presents the interaction of acoustic waves with fiber Bragg and long period gratings; Section 5 discusses some applications developed by the authors, such as the technique for controlling the group delay with flexural waves in fiber gratings, the technique for writing gratings under acoustic excitation and a sensor used for measuring viscosity; finally, Section 6 brings the conclusion and gives some perspectives for the future work in the field. 


\section{Acoustic waves}

An understanding of the propagation of acoustic waves in a solid, circular rod consisting of isotropic, homogeneous materials can be obtained by solving the vector equation of motion that describes the space-time dependent displacement function. One is faced with the solution of a set of three scalar equations in the cylindrical coordinate system from which the displacement field components in the radial, circular and axial directions are obtained [4, 7]. Considering the propagation of a harmonic field, the solution of this set of equations is given as

$$
\begin{aligned}
& u_{r}=U(r)\left\{\begin{array}{l}
\sin (n \theta) \\
\cos (n \theta)
\end{array}\right\} \exp \left[j\left(\omega_{a} t-k_{a} z\right)\right] \\
& u_{\theta}=V(r)\left\{\begin{array}{l}
\cos (n \theta) \\
-\sin (n \theta)
\end{array}\right\} \exp \left[j\left(\omega_{a} t-k_{a} z\right)\right] \\
& u_{z}=W(r)\left\{\begin{array}{l}
\sin (n \theta) \\
\cos (n \theta)
\end{array}\right\} \exp \left[j\left(\omega_{a} t-k_{a} z\right)\right]
\end{aligned}
$$

where $\omega_{a}=2 \pi f_{a}$ and $k_{a}=2 \pi \lambda_{a}$ are the angular frequency and wave number of the acoustic field, respectively, and $n$ is an integer describing the circumferential field behavior. $U(r), V(r)$ and $W(r)$ represent the radial variations, described by Bessel functions [7]. Either the upper or lower set of trigonometric functions in (2) is used to find the desired solutions. The dispersion relation is obtained requiring the stresses to be zero at the cylindrical surface ( $r=a$, with $a$ as the radius of the rod) and choosing an integer value for $n$ in (2). The solution of the resulting characteristic equation delivers the dispersion curves from which the propagation parameters and the behavior of the acoustic phase velocity as a function of the frequency are assessed. A detailed description of the dispersion curves for the cylindrical rod is found in [7].

Different values of $n$ provide solutions for the different modes propagating in the guide. By using the lower set of trigonometric functions in (2) and choosing $n=0$, one obtains longitudinal waves, which shows no angular dependence upon $\theta$ and is axially symmetric, with displacement components in the radial, $r$, and axial, $z$, directions only. By choosing the upper set of trigonometric functions and still keeping $n=0$, one obtains torsional waves, where the displacement components $u_{r}$ and $u_{z}$ vanish, and the remaining component is independent of $\theta$. Finally, flexural waves, which depend on the circumferential angle $\theta$, are obtained by setting $n \neq 0$. In this case, either set of trigonometric functions can be chosen.

The analysis of the dispersion curves of the acoustic phase velocity for each type of acoustic mode is important in order to control the operation of the acousto-optic modulator and obtain the desired effect in the fiber. Initial studies on the propagation of acoustic waves in silica fibers provided the dispersion curves for the propagation of longitudinal, torsional and flexural waves [16]. By using such dispersion curves, it is convenient to work with the normalized frequency, defined as $f_{a} a / c_{t}$, where $f_{a}$ is the acoustic frequency, $a$ is the fiber radius, and $c_{t}$ is the transverse acoustic plane wave velocity in bulk silica $\left(c_{t}=3764 \mathrm{~m} / \mathrm{s}\right)$.

The phase velocity shows a characteristic behavior according to the frequency regime. For instance, in the low frequency regime, when $f_{a} a / c_{t} \ll 1$, only few acoustic modes exist in the guide. For the lowest guided longitudinal mode, the phase velocity approaches that given by the extensional wave in silica $\left(c_{\mathrm{ext}}=5760 \mathrm{~m} / \mathrm{s}\right)$ when $f_{a} a / c_{t} \ll 1$, and it asymptotically assumes the velocity of Rayleigh waves (surface waves) when $f_{a} a / c_{t}>>1$. In this last case, the wave energy is confined to a thin layer within the rod surface, a situation also experienced by torsional and flexural modes. In the high frequency regime, that is, when $f_{a} a / c_{t} \gg 1$, the guide becomes highly multimode, and it is not interesting for acousto-optic interaction, as the existence of many modes makes it difficult to have a proper control of devices. The studies also point out that for acousto-optic interaction to take place there must be a significant overlap area between the acoustic wave and the optical modes in the fiber, which means that 
the regime $f_{a} a / c_{t} \ll 1$ is the most relevant for acousto-optic operation [16].

Considering only the lowest order flexural mode, Blake et al. [17] classified the dispersion behavior according to three different acoustic propagation regimes in the silica fiber. For higher frequencies, where $f_{a} a / c t \gg 1$, the acoustic energy concentrates on the surface of the fiber, and the acoustic wavelength, $\lambda_{a}$, varies as $1 / f_{a}$. For lower frequencies, where $f_{a} a / c t<<1$, the acoustic energy is uniformly distributed over the fiber cross section, and $\lambda_{a}$ varies as

$$
\lambda_{a}=\left(\frac{\pi a c_{\mathrm{ext}}}{f_{a}}\right)^{1 / 2}
$$

where $a$ is the fiber radius, and $c_{\text {ext }}$ is the extensional acoustic wave velocity in silica. Between these two extremes, where $\lambda_{a} \approx 2 \pi a$, there is a transition region. For the acousto-optic interaction to take place in the core of the guide, it is important that the flexural wave operates in the low frequency region. The same analysis can be extended to the operation with the lowest longitudinal mode. By taking the dispersion curves given in [16], a simple calculation shows that the low frequency regime is achieved in conventional single mode fibers $(2 a=125 \mu \mathrm{m})$ when $f_{a}<30 \mathrm{MHz}$. In this case, there exist only three types of modes: the lowest order longitudinal mode, the lowest order torsional mode, and the double degenerated ( $x$ - and $y$-polarized) lowest order flexural mode. It is important to note, however, that the regime classification given above is an approximation, and in fact, the real dispersion curves for the acoustic modes should be taken into account for an exact analysis through the solution of the characteristic equation.

\subsection{Resonances of piezoelectric discs}

Excitation of acoustic waves in fibers is usually done with an acousto-optic modulator. In practice, the modulator is composed of a piezoelectric transducer, shortly named PZT (a disc made, for instance, of lead zirconate titanate) and an acoustic horn (made of glass or metal). Figure 1(a) illustrates one possible configuration in which the optical fiber segment is axially aligned with the horn [18]. Another popular configuration places the piezo-horn device transversally to the fiber $[8,16,17]$. Figure 1(b) shows the piezo disc with its possible vibration directions.

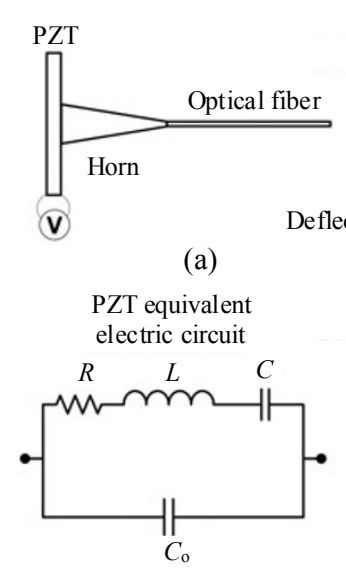

(c)

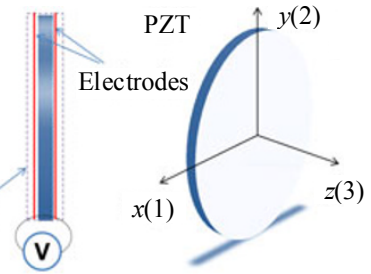

(b) PZT impedance response

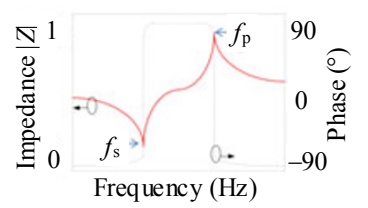

(d)
Fig. 1 Acousto-optic modulator and its parts: (a) schematic of the acousto-optic modulator, (b) PZT in 2-D and 3-D xyz planes, (c) equivalent electric circuit, and (d) impedance-phase response.

When the piezo is driven by an electrical signal applied transversally to the disc, it produces mechanical deformation that is conveyed along the fiber by the horn. Piezoceramic discs present a distinct modal behavior that depends on the excitation frequency, material properties and anisotropy, polarization and geometric dimensions [20]. The commonly used piezoelectric transducers do not respond to any excitation frequency, but only to narrow bands of resonances in which the vibration modes manifest larger and specific mechanical displacements $[19,20,21]$. As the efficiency of the acousto-optic mechanism is strongly dependent on the excitation frequency, and the type of the generated acoustic wave is also determined by the directional deformation of the piezo, the thorough characterization of the transducer response and its resonance modes are necessary for the efficient control and operation of 
acousto-optic devices.

Moreover, the combined analysis of the piezo spectral response and the modal displacement is useful and required for the identification of the acoustic modes coupled to the fiber. Due to the material high dielectric constant and the electric polarization of the electrodes, the transducer can be compared to a capacitor. The element can be represented by an equivalent circuit known as Butterworth-Van Dyke and is illustrated in Fig. 1(c) [22]. The circuit is composed of a $R L C$ series and a parallel circuit $\left(R L C / C_{\mathrm{o}}\right)$, where the resistor $R$, the inductor $L$, and the capacitor $C$ are related to the damping, mass and the elastic PZT constant, respectively. $C_{o}$ is the electric capacitance between the electrodes. Based on this equivalent circuit, a resonator with the angular frequency $\omega=2 \pi f$ can be modeled by using the electrical parameters only. The frequency response of the transducer is then obtained by solving the $R L C$ circuit in terms of the impedance $Z$, written as

$$
Z=R+j\left(\omega L-(\omega C)^{-1}\right) .
$$

For the situation where losses are negligible and the impedance is null, the series resonance $f_{\mathrm{pzt}}$ is obtained from (4) as

$$
f_{s}=(2 \pi \sqrt{L C})^{-1}
$$

A similar deduction applied to the parallel circuit $\left(R L C / / C_{\mathrm{o}}\right)$ in Fig. 1(c) allows the parallel or anti-resonance $f_{p}$ to be obtained

$$
f_{p}=\left(2 \pi \sqrt{L C C_{o} /\left(C+C_{o}\right)}\right)^{-1} .
$$

Once the parameters $R, L$ and $C$ are known or calculated from the material constants, (5) and (6) are used to calculate the piezo resonances and anti-resonances. Figure 1(d) illustrates the transducer spectral response in terms of the impedance magnitude and phase. The PZT resonance frequency $f_{s}$ and anti-resonance frequency $f_{p}$ correspond to the condition of the minimum impedance and maximum impedance, respectively. At these frequencies, the inductance $L$ and the capacitance $C$ cancel each other by making the phase null. As the electrical current flowing across the piezo is related to the mechanical deformation, the reduction in the impedance $Z$ causes a maximum current flow $I$, which is then responsible for the maximum PZT deflection at the resonance $f_{s}$.

Piezo resonances can be measured by impedance-phase methods [23]. Figure 2 shows the measured PZT response in terms of the impedance magnitude and phase response as a function of the excitation frequency. Modes with small damping values, where the transducer losses are minima, present higher amplitudes and sharper resonances. However, higher losses reduce the impedance and phase amplitude of the modes and make the detection of certain resonances more difficult, as can be seen in Fig. 2 from the measured impedance values along the $z$ direction [see Fig. 1(b)].

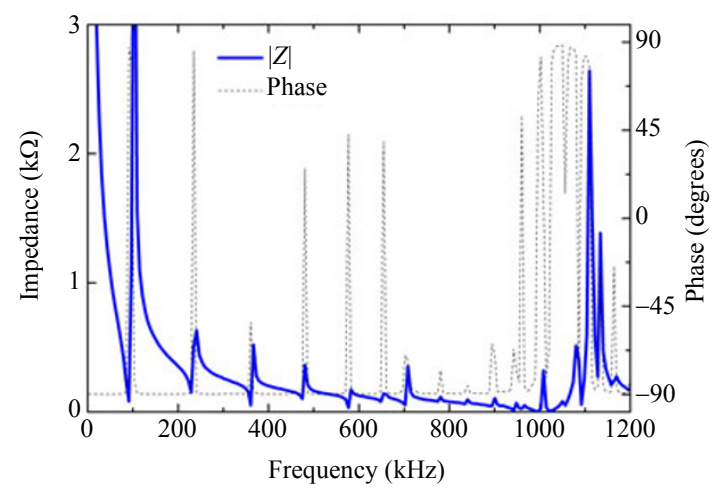

Fig. 2 PZT experimental impedance-phase response (the solid line represents the amplitude, and the dashed line represents the phase response).

However, a more detailed analysis of the coupled electromechanical relations and material constants can be performed by using the finite elements method (FEM). Commercial software tools can be very useful in this case [24, 25]. The FEM requires the discretization of the device geometry in small subdomains (elements) and builds a matrix equation system representing the piezo behavior. Solving the matrix equation allows one to obtain the resonant frequencies and, particularly, the 
mechanical displacements of the piezo disc in its transversal $(z)$ and radial directions $[x$ or $y$ axis in Fig. 1(b)].

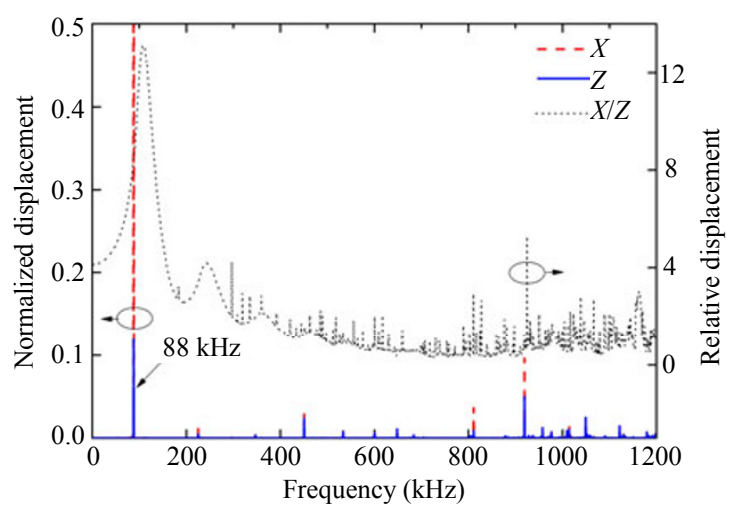

Fig. 3 PZT relative displacements in $x$ and $z$ directions over the frequency, showing the behavior of radial and thickness modes, respectively (the ratio $x / z$ shows the preponderance of the oscillation in a given direction).

The dashed and solid curves in Fig. 3 show the normalized piezo displacements along $x$ (representing changes in the disc diameter) and along $z$ (representing changes in the disc thickness), respectively, over the frequency range up to $1200 \mathrm{kHz}$, obtained through the FEM technique. At $f=88 \mathrm{kHz}$, the fundamental mode has the highest $x$ displacement among all modes. The relative displacement between the $x$ and $z$ directions (taken as the ratio $x / z$ and represented by the dashed curve in Fig. 3) indicates the direction vibration preference (at $f=88 \mathrm{kHz}$, the radial displacement is approximately 16 times larger than the $z$ displacement). The use of such analysis helps the identification of the disc vibration directions that lead to the excitation of flexural waves (for more intense displacements in the radial direction) or longitudinal waves (for more intense displacements in the transversal direction).

\subsection{Excitation of acoustic waves}

Although it is useful, the FEM analysis of the piezo disc is not sufficient to determine the types of acoustic modes excited in the fiber. Knowing that the standard optical fiber (SMF-128), for frequencies below $30 \mathrm{MHz}$, supports only the lowest flexural, torsional and longitudinal acoustic modes, the question on which mode (or modes) is (are) excited depends on the physical arrangement and on the highest displacement of the piezo along the radial or transversal directions at a certain frequency [11].

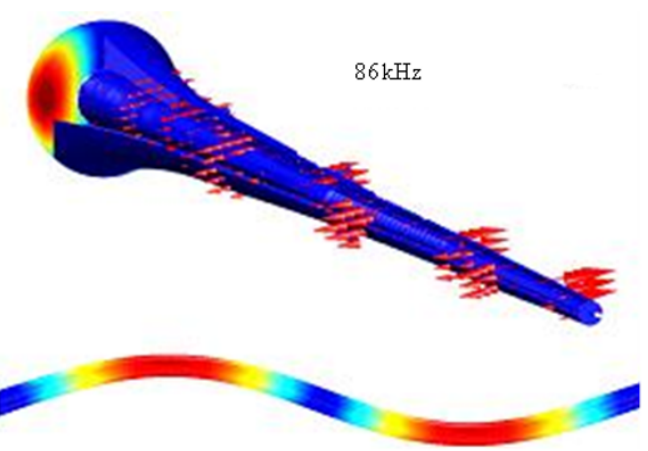

(a)

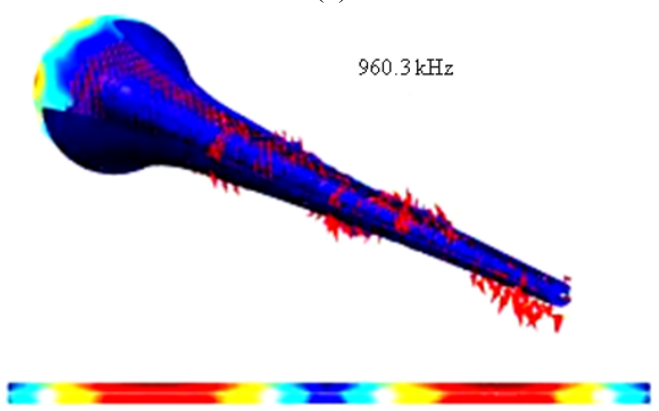

(b)

Fig. 4 FEM simulation of the acousto-optic modulator: (a) the simulated acousto-optic modulator with displacement details of PZT, horn and optical fiber as excited by a flexural wave and (b) the simulated acousto-optic modulator with displacement details of PZT, horn and optical fiber as excited by a longitudinal acoustic wave.

The FEM technique can be of further help and be extended to perform a 3-D simulation of the whole modulator, consisting of the piezo, the horn and the fiber. This indeed helps the identification of the type of the acoustic wave excited in the fiber at the piezo resonances. For instance, Figs. 4(a) and 4(b) show the simulated acousto-optic modulator and details of the piezo disc, horn and optical fiber at the resonance frequencies $f=86 \mathrm{kHz}$ and $960.3 \mathrm{kHz}$, respectively. For instance, the resonance $f=86 \mathrm{kHz}$ 
cause the excitation of a flexural mode, which is characterized by pronounced bends in the fiber, while at $f=906.3 \mathrm{kHz}$ bends are no longer seen and the result is a longitudinal mode, where the displacement component is the highest along the fiber axis. Moreover, from simulations and experiments, it has been observed that the acousto-optic modulator can also excite acoustic waves of complex oscillations, which can be generally termed as hybrid, once they are a composition of flexural, torsional and longitudinal modes.

\subsection{Measurement of acoustic waves}

An extrinsic Fabry-Perot interferometer (EFPI) $[26,27]$ can be used to characterize the acoustic waves propagating in optical fibers [28]. The technique allows the analysis of oscillating surfaces in the space and time domains. Figure 5 shows the basic arrangement. The sensor is composed of a cavity formed by the interface of a cleaved optical fiber (the probe) and the sample under test (the PZT disc surface seen in Fig. 5).

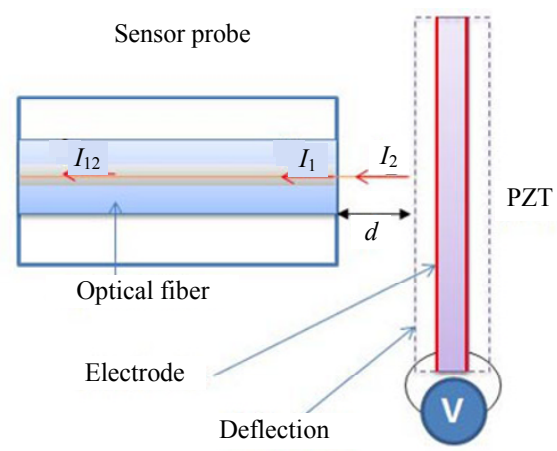

Fig. 5 Diagram of the EFPI optical cavity [28].

A fraction of the light that propagates in the probe toward the sample is partially reflected in the fiber-air interface with the intensity $I_{1}$. The fraction of the light transmitted into the cavity is again reflected on the sample (oscillating) surface with the intensity $I_{2}$. The reflected intensities result in an interference pattern $I_{12}$ that depends on the distance $d$ between the fiber and the sample, and the optical wavelength $\lambda$. The optical intensity modulation caused by the oscillating sample surface is converted into the voltage by a photodetector and is examined with an oscilloscope. The resultant intensity seen by the photodetector is given as

$$
I_{12}=I_{1}+I_{2}+2 \sqrt{I_{1} I_{2}} \cos \left(\frac{4 \pi d}{\lambda}\right)
$$

However, for measurement of flexural and longitudinal waves, the experimental setup of Fig. 6 is used. It consists of two optical sensor heads (probes) based on standard single mode fibers (SMF-28) and optical circulators. The sensor head 1 is used to detect the longitudinal displacement of the fiber, as seen in Fig. 6(b), while the sensor head 2 is used to measure the transversal oscillations (the presence of flexural deformation), as shown in Fig. 6(c). The sensor heads are mounted in a fiber ferrule and fixed in a support mounted in positioning tables. A 50:50 coupler splits the optical power from a tunable laser between the sensor heads 1 and 2. The signals coming from both sensors reach the photodetector, which is connected to an oscilloscope. The measurements with the sensor heads 1 and 2 are not performed simultaneously.

The measurement of flexural acoustic waves and the characterization of bendings are done by using the standing acoustic wave formed when the fiber under test is fixed on its two ends (at the horn tip on one side and the positioning table on the other). This standing wave is represented by

$$
\Psi(x, t)=2 A \cos \left(\frac{2 \pi}{\lambda_{a}} x\right) \sin \left(2 \pi f_{a} t\right)
$$

which describes the spatial and temporal behaviors of the standing wave formed in the fiber and is illustrated in Fig. 6(c) for one oscillation cycle. In (8), $A$ is the amplitude, $\omega_{a}=2 \pi f_{a}$ is the angular frequency, and $k_{a}=2 \pi \lambda_{a}$ is the wave number related to the flexural wavelength $\lambda_{a}$. The expression represents the result of a travelling acoustic wave which is totally reflected at the points where the fiber is fixed, thus forming the standing wave. Figure 6(c) shows that the standing flexural wave 
has zero amplitude at the nodes, which are spaced by the wavelength $\lambda_{a}$.
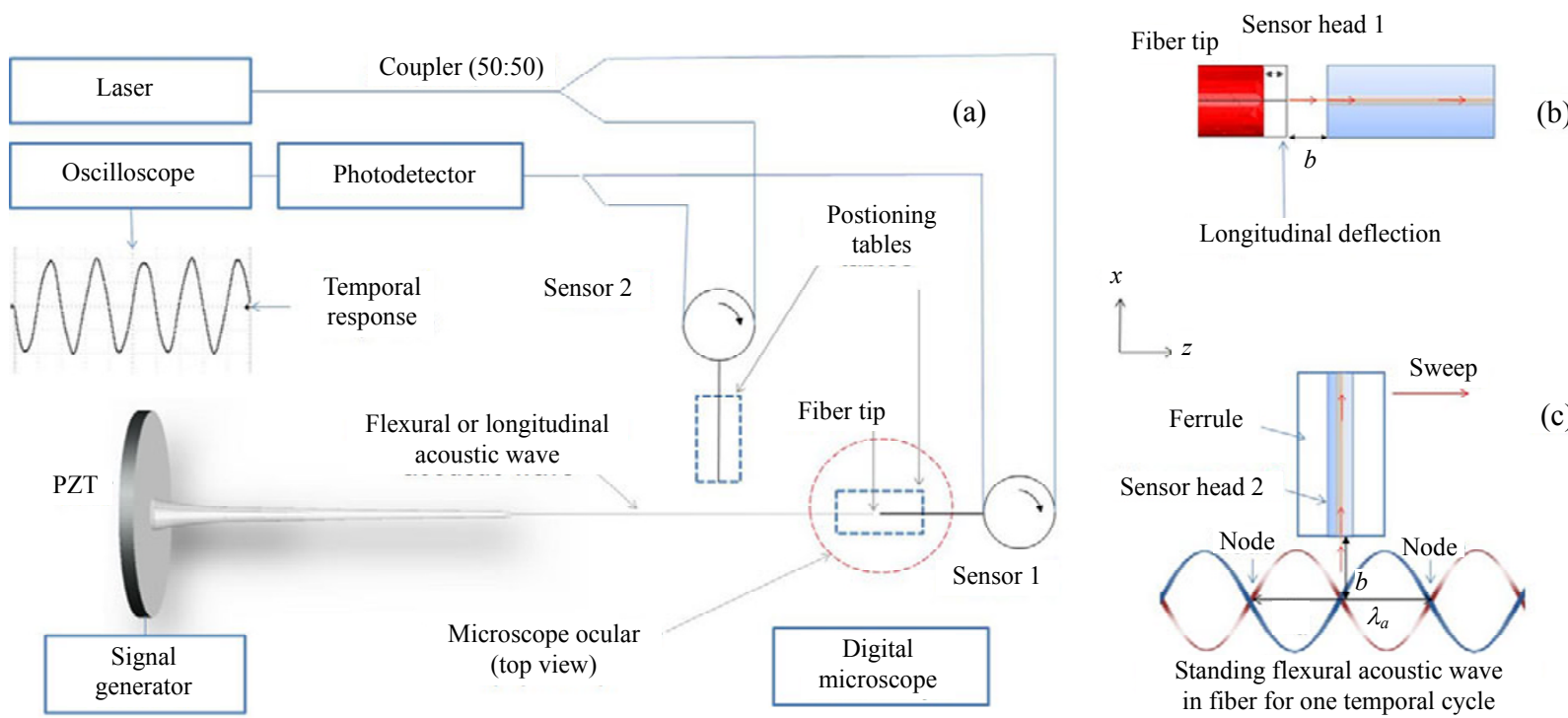

Fig. 6 Test setup for measuring acoustic waves: (a) diagram of the experimental setup used to measure, (b) longitudinal, and (c) flexural acoustic waves in the optical fiber [29].

Once the standing wave is established, the sensor head 2 either is set to measure the temporal periodic oscillation at an arbitrary position along the fiber (with the exception of points located at the wave nodes, where the oscillation is zero) or is swept over the fiber axis to measure the troughs and crests of the flexural wave. One measures the acoustic frequency in the first approach and in the second the peak-to-peak amplitude (acoustic wavelength, $\lambda_{a}$ ) of the flexural mode. Based on the measured wavelength $\lambda_{a}$ and frequency $f_{a}$, it is possible to estimate the phase velocity through the

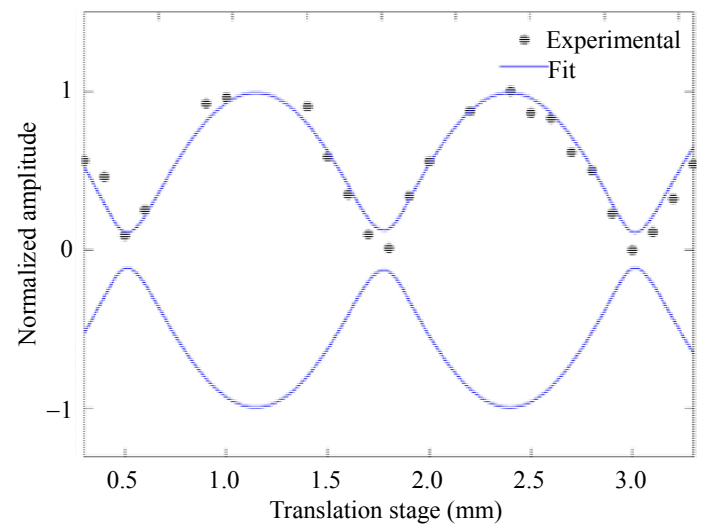

Fig. 7 Spatial behavior of the standing flexural wave in the optical fiber, measured by translating the probe over the fiber surface [see Fig. 6(c)] [28]. relationship given in (3) for the low-frequency propagating regime. Figure 7 shows the measured (normalized) amplitude of the standing flexural acoustic wave formed in the fiber at $f=176.7 \mathrm{kHz}$ when the sensor head 2 is swept over a distance of more than $3 \mathrm{~mm}$ along the modulator axial direction. Applying a fitting to the measured points provides the curve from which the flexural wavelength is calculated (in this case, $\lambda_{a}=2.50 \mathrm{~mm}$ ).

On the other hand, when longitudinal acoustic waves are excited, the fiber is stretched at its tip [see Fig. 6(b)], making it possible to measure the acoustic frequency $f_{a}$ by assessing the changes in the optical cavity, given by the distance $d$, formed by the fiber tip at its loose end (in this case, the fiber end located at the positioning table must be set free to oscillate) and the sensor head 1 [29]. Additionally, in order to measure the acoustic frequency, the detected periodic signal must be converted into the frequency domain by using the oscilloscope fast Fourier transform (FFT) function. On performing the measurement, one must verify that no flexural deformation (or a minimum) takes place. This is accomplished by sweeping the sensor head 2 over the fiber axial length. By this way, the maximum 
amplitude detected by the sensor head 1 is related to the resonant frequency of the longitudinal acoustic wave only. However, the presence of flexural deformation measured by the sensor head 2 means that flexural waves also exist in the fiber leading to the presence of hybrid modes.

Figure 8 shows the spectrum of longitudinal waves excited at $f_{a}=655 \mathrm{kHz}, 955 \mathrm{kHz}$, and $1002 \mathrm{kHz}$. At $f_{\mathrm{a}}=655 \mathrm{kHz}$, harmonics are also observed in the oscilloscope on both sides of the fundamental resonance. This is due to the superposition of residual flexural deflections, which remains present in the fiber. The resonances at $f_{\mathrm{a}}=$ $955 \mathrm{kHz}$ and $1002 \mathrm{kHz}$ show that only the axial stretching of the fiber takes place. Taking these measured frequencies and considering the extensional acoustic velocity as $c_{\text {ext }}=5760 \mathrm{~m} / \mathrm{s}$ in bulk silica, the acoustic wavelength for the three axial modes are estimated as $\lambda_{a}=8.79 \mathrm{~mm}, 6.03 \mathrm{~mm}$ and $5.75 \mathrm{~mm}$, respectively, considering the relationship of the phase velocity, $c_{\text {ext }}=\lambda_{a} f_{a}$, for the propagation in the high frequency region.

In summary, from measurements and simulations, it is has been observed that the particular acousto-optic modulator under test supports a large variety of flexural acoustic modes in the range between $1 \mathrm{kHz}$ and $250 \mathrm{kHz}$ and mostly longitudinal modes in the range between $600 \mathrm{kHz}$ and $1000 \mathrm{kHz}$. The range in-between is mostly characterized by flexural and hybrid modes.

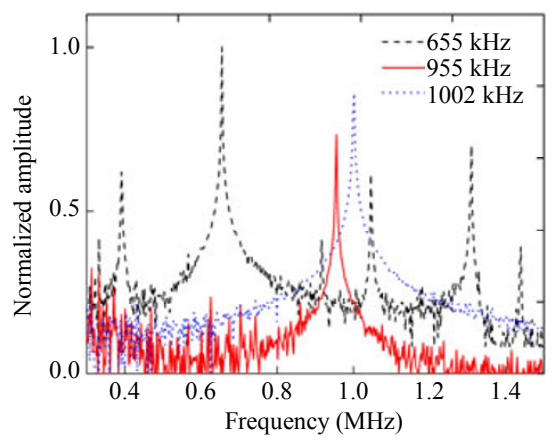

Fig. 8 Spectrum response seen in the oscilloscope of longitudinal acoustic waves excited at $f_{a}=655 \mathrm{kHz}, 955 \mathrm{kHz}$ and $1002 \mathrm{kHz}$ [29].

\section{Acousto-optic interaction in fibers}

The loss of power of propagating modes due to microbend effects in optical fibers is a well-known phenomenon. This occurs due to the coupling of guided to radiation modes in the cladding when phase-matching exists. Textbooks on optical fibers provide a basic understanding and description of such a coupling mechanism [30]. A thorough study of mode coupling between the $L P_{01}$ and radiation modes or between the $L P_{01}$ and the $L P_{11}$ modes due to very small bends (microbends) is found in [31]. In this work, Taylor proposed the generation of microbends in fibers using mechanical transducers to produce a periodic perturbation in the direction of the fiber axis, which can be used as a $L P_{01} \leftrightarrow L P_{11}$ mode converter.

The employment of acoustic waves in fibers to generate microbends was initially proposed by Engan et al. [14], Kim et al. [32] and Blake et al. [17]. In their work, flexural acoustic waves were used to generate microbends and achieve mode conversion in a two-mode optical fiber using an acousto-optic modulator. Considering the coupling between two optical modes, and denoting mode 1 as the fundamental mode, which contains all the energy before the acousto-optic interaction, and mode 2 as the one that carries a portion of the optical power after the interaction takes place, the fraction of the light coupled from mode 1 to mode 2 over the interaction length $\left(L_{b}\right)$, is given as [33]

$$
\eta=\frac{|\kappa|^{2}}{|\kappa|^{2}+\left(\frac{\Delta \beta}{2}\right)^{2}} \sin ^{2}\left\{\left[|\kappa|^{2}+\left(\frac{\Delta \beta}{2}\right)^{2}\right]^{1 / 2} L_{b}\right\}
$$

with $\kappa$ as the acousto-optic coupling coefficient given as

$$
\kappa=\frac{k}{2} \int_{A} \Delta n(x, y) E_{1}(x, y) E_{2}(x, y) d x d y
$$

where $k$ is the optical wave number, $E_{1}$ and $E_{2}$ are the transversal field distributions of the optical modes 1 and 2, respectively, $\Delta n$ is the refractive index change due to the acousto-optic effect (the 
sum of contributions due to the material index change through the elasto-optic effect and due to the optical path length change caused by the microbends), and $\Delta \beta$ is the phase difference, defined as

$$
\Delta \beta=2 \pi\left(\frac{1}{L_{i}(\lambda)}-\frac{1}{\lambda_{a}\left(f_{a}\right)}\right)
$$

where $\lambda_{a}$ is the wavelength of the acoustic wave, $L_{i}$ is the intermodal beat length, defined as the necessary length for mode 1 to acquire a phase shift of $\Delta \varphi=2 \pi$ in relation to mode 2. From (9), if $\Delta \beta$ is much larger than $|\kappa|$ in the denominator, almost no light is coupled from mode 1 to mode 2 , while if $\Delta \beta$ $=0$, all light is coupled to mode 2 . The intermodal beat length is calculated as

$$
L_{i}=\frac{\pi}{2|\kappa|} .
$$

From (11), one notes that when $\Delta \beta=0$ the phase matching is satisfied, and for this particular case

$$
L_{i}(\lambda) \equiv \frac{\lambda}{n_{1}(\lambda)-n_{2}(\lambda)}=\lambda_{a} .
$$

This means that for obtaining the total power conversion from one mode to the other, the acoustic wavelength must be equal to the beat length between the modes. For standard single-mode fibers operating in the range from $1300 \mathrm{~nm}$ to $1600 \mathrm{~nm}$, the estimation of beat lengths gives values varying from almost $500 \mu \mathrm{m}$ to $650 \mu \mathrm{m}$ [34]. By this way, for optimal mode coupling using standard fibers, the acoustic wavelength must also lie in this range, which is much shorter than the wavelength of the flexural mode excited at lower frequencies and discussed in Section 2.3.

Since these initial studies, many other works [35-37] followed, in which the design and operation of optical couplers, filters, gain equalizers have been demonstrated. In all these devices, flexural waves have been excited by using the configuration where the horn was transversally placed in relation to the fiber axis. Moreover, as a way of enhancing the strength of the acousto-optic interaction, tapers have also been used. This was done in order to reduce the fiber diameter and therefore enhance the strength of the flexural excitation.

\section{Interaction with gratings inscribed in fibers}

Another important contribution to the area of acousto-optics comes from the interaction of acoustic waves with gratings inscribed in optical fibers. Gratings are versatile and useful devices employed in several sensing and telecommunications applications [38, 39]. A grating can be inscribed in the fiber through several methods and is formed by the periodical change in the refractive index and stress-optic effect along the fiber core. Gratings are characterized by three main parameters: reflectivity/transmissivity, period and length, which can be modified by the strain field generated by the propagation of the acoustic field in the fiber. Indeed, this mechanism provides another degree of flexibility, offering new modulating and control alternatives and extending the range of applications for all-fiber grating devices.

The following sections will provide an understanding of the interaction of longitudinal and flexural waves with Bragg and long period gratings through the acousto-optic mechanism in an attempt to cover their application in several useful techniques and devices.

\subsection{Interaction of FBGs with longitudinal waves}

When an acoustic longitudinal wave propagates along the fiber, it causes a periodic strain field, generating compression and rarefaction zones in the material. By means of the elasto-optic mechanism, the strain promotes a change in the fiber effective refractive index. If a grating is inscribed in the fiber core, it will also be subjected to the strain, which will not only cause an additional change in the effective indices of the forward and backward propagating optical modes in the guide, but also modulate the grating pitch. This modulation causes the appearance of additional bands on both sides of 
the grating reflection spectrum with regularly spaced peaks, which are originated by the coupling of the counter-propagating optical modes, resulting from the fine pitch Bragg grating, due to the coarse acoustical pitch produced by the longitudinal wave. This mechanism is known as superlattice modulation in fiber gratings, and the structure formed in the interaction is known as super grating or sampled grating. Sampled gratings generated by acoustical waves were first reported in [40].

Russell and Liu [41] developed a simple mathematical model to describe the appearance of side bands. The periodic strain field of the acoustic wave perturbs the grating in two ways: (1) by increasing the average refractive index due to changes in the optical path length and the elasto-optic mechanism and (2) by periodically modulating the grating pitch, causing spatial-frequency modulation. The total change in the effective refractive index, experienced by the grating, is given by the strain field and the contribution of the pitch modulation and can be calculated as

$$
\begin{aligned}
& \Delta n_{\mathrm{eff}}=(1-\chi) \varepsilon(z, t)+ \\
& n_{0}\left\{1+C \cos \left[K\left(z-\int \varepsilon(z, t) d z\right)\right]\right\}
\end{aligned}
$$

where

$$
\varepsilon(z, t)=\varepsilon_{0} \cos \left(k_{a} z-\omega_{a} t\right)
$$

with $k_{a}=2 \pi / \lambda_{a}$ as the acoustic wave vector. $\lambda_{a}$ is the acoustic wavelength, $\omega_{a}$ is the angular acoustic frequency, and $\chi$ is the magnetic susceptibility of the fiber. $C$ is the modulation depth of the effective refractive index $n_{0}$ in the unperturbed grating, $K=$ $2 \pi / \Lambda$ is the optical grating vector, with $\Lambda$ being the unperturbed grating pitch. $\varepsilon_{0}$ is the peak strain of the wave and depends on the area of the acoustic beam, the carried acoustic power in a medium with Young's modulus $E$ and the acoustic group velocity $v_{g a}$ [41]. By replacing (15) in (14), it is shown that the pitch modulation produces a sequence of sidebands in the spatial frequency domain whose amplitudes can be described by a standard Bessel function expansion [41], as follows

$$
\begin{aligned}
& \cos [K z-r \sin \varepsilon(z, t)]= \\
& J_{0}(r) \cos K z+\sum_{n=1}^{\infty} J_{n}(r)\left\{(-1)^{n} .\right. \\
& \cos \left[\left(K+n k_{a}\right) z-n \omega_{a} t\right]+ \\
& \left.\cos \left[\left(K-n k_{a}\right) z+n \omega_{a} t\right]\right\}
\end{aligned}
$$

where $r$ is the ratio $\left(K \varepsilon_{0} / k_{a}\right)$. This formulation leads to a sequence of ghosts of the original fiber grating forming at spatial frequencies given by successive spatial sidebands of $K$. Figure 9 pictorially shows the situation of the perturbed grating and the corresponding optical response.

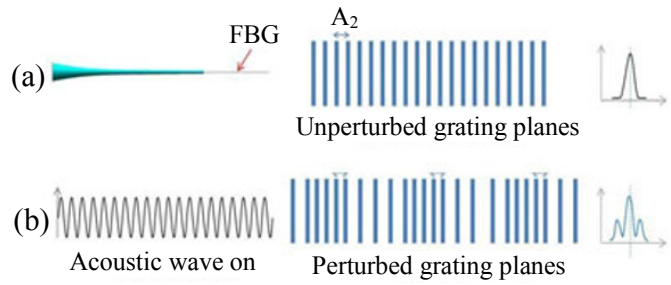

Fig. 9 Representation of the sample modulation produced by the longitudinal acoustic wave upon interaction with the fiber Bragg grating (FBG).

However, a numerical approach for examining the interaction of the longitudinal acoustic mode and the FBG taking into account the dimensions of the piezo-horn-fiber setup is required. This approach can be performed by using the FEM [42] and the transfer matrix method (TMM) [43, 44]. The FEM approach allows the strain field caused by the acoustic wave to be completely characterized along the structure while the TMM is used to obtain the optical spectrum of the corresponding strained grating. The FEM is useful because it takes into account the strain in the horn-fiber setup, considering the real dimensions of the acousto-optic modulator [45]. The FEM and TMM approaches are adequate and accurate methods, particularly if the structure under analysis presents an arbitrary cross-sectional shape along the fiber axis.

The methodology consists of two steps. Firstly, the strain field in the whole structure (horn, taper and FBG) is obtained by using the FEM. Secondly, the calculated strain field is used in the TMM 
algorithm to obtain the FBG spectrum. Figure 10 shows the 1-D discretization of the acousto-optic modulator. The structure is composed of $N$ one-dimensional elements with the length $\Delta z=L_{D} / N$ separated by nodes, where $L_{D}$ is the total length of the modulator. Each element is associated with a value that represents the area of the structure at that section.

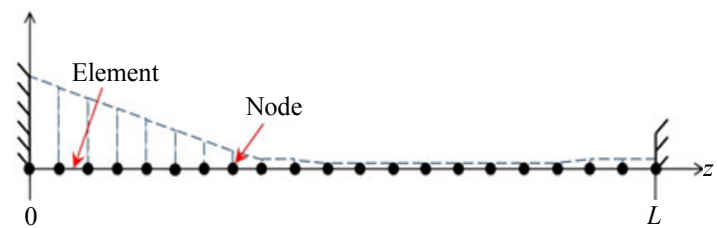

Fig. 10 Diagram of the 1-D discretization of the acousto-optic modulator for the simulation with the FEM [45].

The differential equation of the motion that represents the propagation of the acoustic wave in the structure is given by

$$
E \cdot \frac{\partial}{\partial z}\left(A(z) \frac{\partial u(z, t)}{\partial z}\right)-\rho \cdot A(z) \frac{\partial^{2} u(z, t)}{\partial t^{2}}=0
$$

where $u$ is the axial displacement, which is dependent on the position $z$ and on the time $t$, and $\partial u / \partial z$ is the longitudinal strain $\varepsilon$. The term $A(z)$ accounts for the variable size of the structure along the $z$ axis. $E$ is the Young modulus, and $\rho$ is the silica density, assumed to be $72.5 \mathrm{GPa}$ and $2200 \mathrm{~kg} / \mathrm{m}^{3}$ for the silica, respectively. In the analysis, the damping of the acoustic wave in the structure is neglected. The external excitation $P(t)$ is applied as the combination of a constant load $\left(P_{\mathrm{DC}}\right)$ and a harmonic load of the frequency $\omega$ and amplitude $P_{0}$ generated by the piezo element. A classical linear approach for the finite elements is employed. After the one-dimensional discretization, the final matrix form of the problem is given by

$$
\mathbf{M u}+\mathbf{K u}=\mathbf{P} .
$$

In this expression, $\mathbf{M}$ and $\mathbf{K}$ are the mass and stiffness matrices of the structure, obtained by the superposition of mass and stiffness matrix of each element of the structure and given by

$$
\left[M_{i j}^{e}\right]=\frac{\rho A^{e} \Delta z}{2}\left[\begin{array}{ll}
1 & 0 \\
0 & 1
\end{array}\right]
$$

and

$$
\left[K_{i j}^{e}\right]=\frac{A^{e} E}{\Delta z}\left[\begin{array}{cc}
1 & -1 \\
-1 & 1
\end{array}\right],
$$

respectively, where the superscript $e$ represents each element with particular properties. Furthermore, u and $\ddot{\mathbf{u}}$ in (18) represent the nodal displacement and acceleration vectors, respectively. The vector $\mathbf{P}$ is the nodal generalized force and is null, except for the first component, associated with the node at $z=$ 0 . The external load is assumed as being a concentrated point load at the tip of the silica horn. Considering an excitation of the form $\mathbf{P}=\mathbf{P}_{\mathrm{DC}}+$ $\mathbf{P}_{0} \exp (j \omega t)$, where $\mathbf{P}_{\mathrm{DC}}$ is a constant load vector, and $\mathbf{P}_{0}$ is the amplitude of the harmonic load vector. Since the system is assumed linear, the solution of this problem can be found by solving the following equations:

$$
\begin{gathered}
\mathbf{M} \ddot{\mathbf{u}}_{\mathrm{DC}}+\mathbf{K} \mathbf{u}_{\mathrm{DC}}=\mathbf{P}_{\mathrm{DC}} \\
\mathbf{M} \ddot{\mathbf{u}}_{t}+\mathbf{K} \mathbf{u}_{t}=\mathbf{P}_{0} \exp (j \omega t)
\end{gathered}
$$

where $\mathbf{P}_{\mathrm{DC}}$ can be understood as a preload component. In this case, the acceleration is null, and (21) can be simplified to

$$
\mathbf{u}_{\mathrm{DC}}=\mathbf{K}^{-1} \mathbf{P}_{\mathrm{DC}} \text {. }
$$

On the other hand, since the time dependent load is harmonic, the solution for (22) has the form $\mathbf{u}_{t}=\mathbf{u}_{0} \exp (j \omega t)$. After replacing it in (22) one obtains

$$
\left(-\omega^{2} \mathbf{M}+\mathbf{K}\right) \mathbf{u}_{0}=\mathbf{P}_{0} .
$$

The displacement vector solution $\mathbf{u}_{0}$ is highly dependent on the acoustic excitation (amplitude $\mathbf{P}_{0}$ and frequency $\omega_{a}$ ). Thus, the final solution will be given by

$$
\mathbf{u}=\mathbf{u}_{\mathrm{DC}}+\mathbf{u}_{0} .
$$

Once the displacement field is obtained, the strain field in each finite element is found by differentiation, inside each finite element, as

$$
\varepsilon^{e}=\frac{u^{e+1}-u^{e}}{\Delta z} \text {. }
$$

In this case, as the finite element is linear, $u^{e+1}$ and $u^{e}$ are the displacements in the local nodes $e^{+1}$ and $e$, respectively. 
On the other hand, the TMM is derived from the coupled mode theory. Since this is a well-known method [43], its description will be omitted here. The formalism used in the algorithm is based on [39]. The fiber grating is divided in piecewise-uniform sections, and the final solutions for each section are combined multiplying the matrices associated with each section, from which the amplitude and power reflection coefficients of the grating optical response are calculated.

The TMM approach is applied to obtain the optical response of different grating designs. In such approach, the effective refractive index perturbation in the core is described by [39]

$$
\delta n_{\mathrm{eff}}(z)=\delta n_{0}(z)\left[1+v \cos \left(\frac{2 \pi}{\Lambda} z\right)\right]
$$

where $\delta n_{0}$ is the average refraction index change along the fiber axis, $v$ is the fringe visibility, and $\Lambda$ is the grating nominal pitch. The presence of the grating imposes a dielectric perturbation to the waveguide and forces the coupling between the fundamental forward and backward propagating optical modes. However, the propagating acoustic wave turns the grating pitch non-uniform and changes the path of the optical mode, as evidenced by the refractive index change in (14). Taking into account this non-uniformity, the reflection and transmission spectra can still be calculated by considering the same piecewise approach, whereby the grating is divided into discrete uniform sections that are individually represented by a matrix. The solution is found by multiplying the matrices associated with each one of the sections. When the characteristic equation is solved by making the matrix determinant equal to zero, the resulting polynomial enables the eigenvalues to be found [43].

The connection between the FEM and TMM approaches occurs over the relationship that computes the variation of the design wavelength along the fiber axis (z-axis) in the TMM as a function of the strain field [39]. This relationship is given by

$$
\lambda_{D}(z)=\lambda_{D 0}+\left(1+\left(1-p_{e}\right) \varepsilon(z)\right)
$$

where $p_{e}$ is the photoelastic coefficient, and $\varepsilon(z)$ is the strain field calculated using (26) in the FEM. By this way, once the strain field is known along the fiber and particularly along the grating, one can calculate the grating optical response (reflected and transmitted spectra) [45].

Figure 11 shows a comparison of the experimental response with the simulated response of a fiber Bragg grating to which an acoustic excitation at $f=1021 \mathrm{MHz}$ and $V_{\mathrm{PZT}}=10 \mathrm{~V}$ (corresponding to an estimated $P_{0}=1 \mathrm{~N}$ applied to the base of the silica horn) is applied.

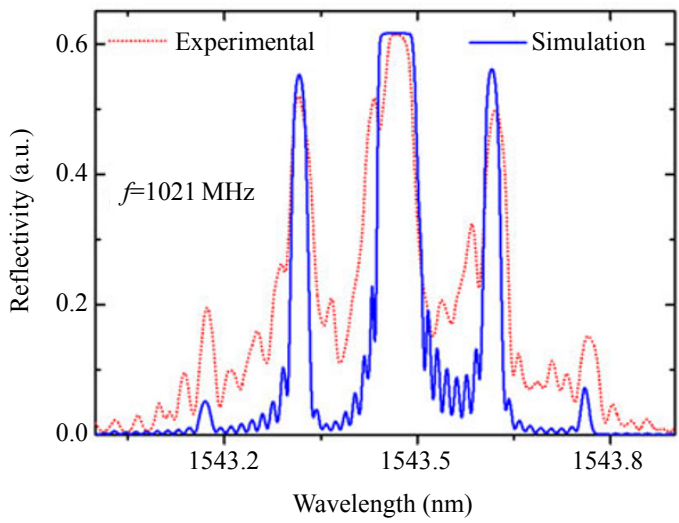

Fig. 11 Experimental and simulated responses (with the FEM and TMM) of a fiber Bragg grating to which an acoustic excitation at $f=1021 \mathrm{MHz}$ is applied [18].

\subsection{Interaction of flexural waves with FBGs}

Sections 2.2 and 2.3 described how flexural, longitudinal or hybrid acoustic waves are generated and measured. The rod-like structure represented by the standard silica fiber supports the lowest order flexural, torsional and longitudinal modes. However, the existence of a specific mode in the guide depends on the excitation condition. The FEM modal analysis is useful for the identification of the different acoustic modes in the structure, whose results corroborates experimental observations. For instance, in the low frequency regime (up to $250 \mathrm{kHz}$ ) 
most resonant modes generate flexural acoustic waves that cause strong bending of the fiber. Even though they are dominant, the lowest order longitudinal mode is always present, but with a weaker amplitude. The result is the excitation of a hybrid wave. The joint effect of the resulting strain due to both waves leads to the generation of a chirp in the Bragg grating, as observed in its reflection response, as seen in Fig. 12.

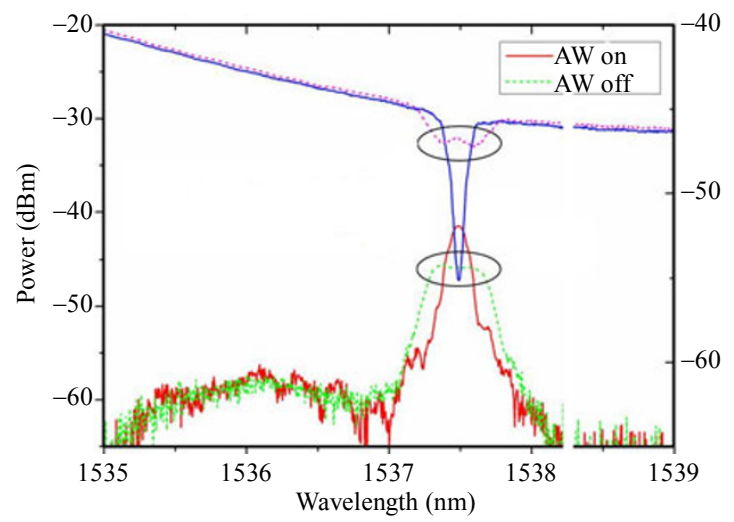

Fig. 12 Chirp generated by a flexural acoustic wave excited at $f=80 \mathrm{kHz}$ (the curves show the $\mathrm{FBG}$ reflected and transmitted spectra before and after the acoustic wave is turned on) $[46]$.

The consequence of such an interaction is that by changing the voltage applied to the piezo disc one is able to control the chirp amount applied to the grating. The result can be better seen in Fig. 13 .

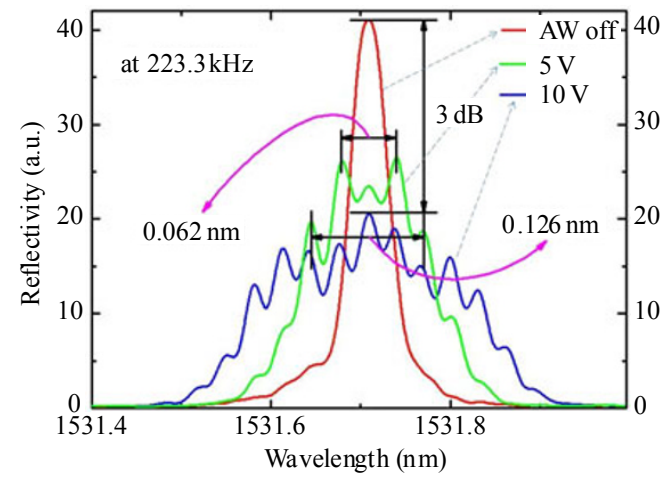

Fig. 13 FBG reflection spectrum when excited at $f=$ $223.3 \mathrm{kHz}$ (the ripples seen on the contour of the waveform may also indicate the presence of a longitudinal acoustic wave) [18].

The chirp effect is also accompanied by a very small shift in the Bragg peak wavelength, with the order of $20 \mathrm{pm}$ [18]. This mechanism has been early observed in gratings inscribed in microstructured fibers [46], but has been observed as well in gratings written in standard ones [18].

\subsection{Interaction of acoustic waves with LPGs}

A long period grating (LPG) is an optical fiber grating, whose period is chosen in order to couple light from the fundamental guided mode to forward propagating cladding modes. Once the energy of the cladding modes is lost due to absorption and scattering in the surrounding environment, a rejection band is measured in the transmission spectrum [47]. The wavelength-dependent phase-matching condition in LPGs is governed by the relationship

$$
\lambda^{m}=\left(n_{\mathrm{co}}-n_{\mathrm{cl}}^{m}\right) \Lambda
$$

where $\lambda^{m}$ is the peak wavelength of the $m$ th attenuation band, $n_{\mathrm{co}}$ and $n_{\mathrm{cl}}^{m}$ represent the effective indices of the fundamental guided mode and the $m$ th $L P_{0 m}$ cladding mode, respectively [48], and $\Lambda$ is the grating period, which, in contrast to fiber Bragg gratings, ranges in the hundreds of micrometers due to the small differences between the effective refractive indices of the core and cladding modes [49]. The minimum transmission of the rejection band, $\lambda^{m}$, is calculated as

$$
T^{m}=1-\sin ^{2}\left(\kappa_{m} L\right)
$$

where $L$ is the length of the LPG, and $\kappa_{m}$ is the coupling coefficient for the $m$ th cladding mode, which is determined by the overlap integral of the core and cladding modes and by the amplitude of the periodic modulation of the mode propagation constants [48].

When an acoustic wave interacts with the LPG, the strain $\varepsilon(z)$ generated by the wave shifts the LPG rejection band. This shift is computed as

$$
\frac{\Delta \lambda^{m}}{\lambda^{m}}=\left(1+p_{e}\right) \varepsilon(z)
$$

where $\Delta \lambda^{m}$ is the wavelength shift experienced by the grating, $\lambda^{m}$ is the peak wavelength of the 
attenuation band when the fiber is at rest, and $p_{e}$ is the photoelastic coefficient which is assumed to be $\left(-1.191 \times 10^{-6}\right) \varepsilon^{-1}$ for an LPG [50]. The same numerical approach described in Section 4.1 can be used to simulate the acousto-optic interaction. For instance, either the FEM or an alternative technique (such as the method of the assumed mode [51, 52]) can be used to calculate the strain field. The result is passed over to the TMM via the "design wavelength" in (28). This is achieved by using the relationship in (30), considering an infinitesimally weak grating with the period $\Lambda$ [43, 45], and making $\Delta \lambda^{m}=\Delta \lambda_{D}$ and $\lambda^{m}=\lambda_{D 0}$, where $\lambda_{D 0}$ is the peak wavelength of the rejection band when the fiber is at rest. By this way, both the effect of the period variation and the change in the LPG effective refractive index can be accounted for at each point inside the grating.

Figure 14 shows experimental results for the excitation of the LPG at $f=60.6 \mathrm{kHz}$. The PZT load varies from $V_{\mathrm{PZT}}=0$ to $10 \mathrm{~V}$. The increase in the amplitude of the acoustic wave reduces the peak transmissivity of the LPG attenuation band and induces a shift to longer wavelengths ("red shift").

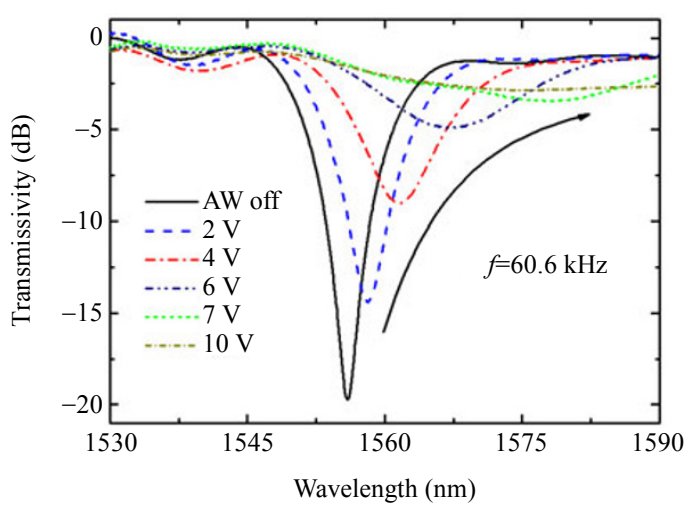

Fig. 14 LPG spectrum behavior when the PZT load varies from 0 to $10 \mathrm{~V}$ at $f=60.6 \mathrm{kHz}$ [52].

Figure 15 shows the LPG peak transmissivity behavior when the PZT load changes (also is related to the force applied to the horn) at the excitation frequency $f=60.6 \mathrm{kHz}$. At $V_{\mathrm{PZT}}=10 \mathrm{~V}$, the force delivered by the PZT is $F=0.0042 \mathrm{~N}$ (estimated through simulations) and a about $17 \mathrm{~dB}$ decrease in the attenuation peak is achieved. Moreover, the relationship between the amplitude of the acoustic wave and the wavelength shift is quasi-linear up to $6 \mathrm{~V}$. For this case, a wavelength shift in $\Delta \lambda=$ $31.6 \mathrm{~nm}$ over the same voltage range is observed [52].

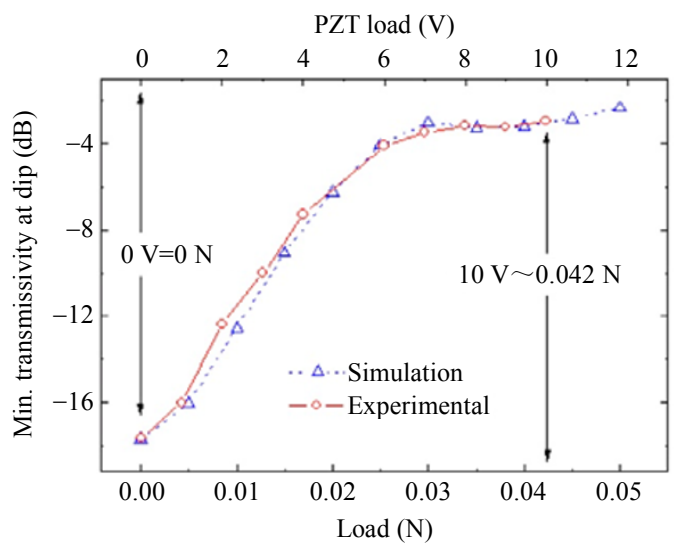

Fig. 15 Experimental and simulation results for the behavior of the LPG peak transmissivity versus the PZT load (in voltage) with the corresponding force (in Newtons) [52].

Excitation of LPGs at higher frequencies, where the predominant acoustic mode is longitudinal [11], leads to no effects in the LPG spectrum. This behavior indicates then that changes in the LPG characteristics are mainly due to the geometric contribution (bends) of the flexural wave to changes in the effective refractive index through the elasto-optic mechanism.

\section{Applications}

Since the first demonstration of the acousto-optic mechanism in fibers, several applications have been developed. They can basically be classified as applications that use only acoustic waves and fibers and applications where the acoustic waves interact with fiber gratings. Examples of the first class are found in $[32,33,36]$. Examples of the second class are found in [53-62]. The three reported examples below are taken from developments of the authors in recent years and 
constitute new applications of the acousto-optic effect that can be potentially used in the fields of sensing and optical communications.

\subsection{Dispersion compensation}

Chromatic dispersion management is an important issue for a long-haul transmission system especially for communication rates of $40 \mathrm{~Gb} / \mathrm{s}$ or above. The use of FBGs for dispersion compensation in place of the dispersion compensation fiber $[63,64]$ is an alternative solution as it reduces insertion losses, avoids nonlinearity and reduces costs [65-67]. FBGs can compensate both first and second order dispersion using nonlinear chirped fiber gratings, but the special chirped phase mask or complicated methods are required in their fabrication. At the same time, the control of the residual dispersion in optical links of high bit rates is even more critical, and a dynamic per-channel dispersion trimming is required [68-70]. In this case, the standard approach for the dynamic dispersion compensation employs chirped FBGs based on a thermal tuning technique [68], which is a very slow process. However, the dynamic control of the dispersion parameter can also be achieved using the acousto-optic effect, which is a faster mechanism, presenting a response time of approximately $17 \mu \mathrm{s}$ in silica waveguides, making it useful for the control of group delay and pulse shaping devices. The acousto-optic effect leads to the broadening of the FBG when flexural waves interact with the grating, changing its group delay and dispersion parameter [46]. One interesting aspect is that this tunable dispersion compensator can be developed using a uniform fiber Bragg grating as opposed to many current FBG compensators that use chirped gratings.

The configuration for such compensator consists of the silica horn-fiber system, with an optical fiber containing the uniform FBG. In the description that follows, the uniform FBG is inscribed in a standard single mode photosensitive fiber by direct writing, using an exciplex $\mathrm{KrF}$ laser. The total length of the grating is $L_{g}=25 \mathrm{~mm}$. By adjusting the excitation frequency, the grating group delay profile as well as its bandwidth can be changed. Figure 16 shows the reflection and group delay behavior when different acoustic frequencies (flexural waves) excite the grating at $f=58 \mathrm{kHz}, 117 \mathrm{kHz}$ and $220 \mathrm{kHz}$, respectively.

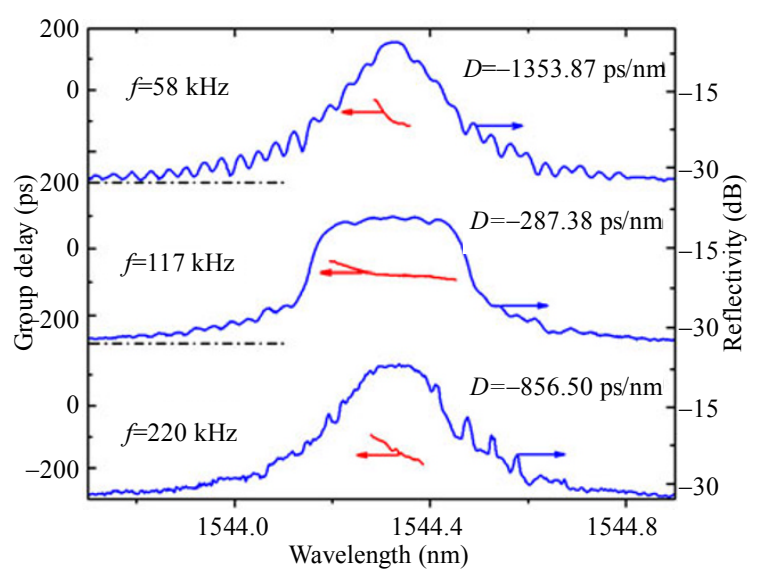

Fig. 16 FBG spectrum and group delay behavior when a flexural acoustic wave is set at $f=58 \mathrm{kHz}, 117 \mathrm{kHz}$ and $220 \mathrm{kHz}$, respectively [18].

The dispersion coefficient is estimated from the resultant group delay measurement. In this case, the dispersion varies from $-1353.87 \mathrm{ps} / \mathrm{nm}$ at $f=58 \mathrm{kHz}$ over $-287.38 \mathrm{ps} / \mathrm{nm}$ at $f=117 \mathrm{kHz}$ to $-856.60 \mathrm{ps} / \mathrm{nm}$ at $f=220 \mathrm{kHz}$. By fixing the excitation frequency, one can also change the group delay and the grating bandwidth through the amplitude of the acoustic wave. Experimental observations have revealed that the group delay at $f=117 \mathrm{kHz}$ presents the most linear behavior in the range between $\lambda=1544.25 \mathrm{~nm}$ and $1544.46 \mathrm{~nm}$. This frequency is then selected for the control of the group delay by varying the PZT load. Figure 17 shows the linear dispersion behavior when the fiber is acoustically excited at $f=117 \mathrm{kHz}$. The variation of the piezo load shows that the dispersion parameter can be controlled in the range from ca. $-550 \mathrm{ps} / \mathrm{nm}$ (at $V_{\mathrm{PZT}}=6 \mathrm{~V}$ ) to $-130 \mathrm{ps} / \mathrm{nm}$ (at $V_{\mathrm{PZT}}=10 \mathrm{~V}$ ) [18]. The experiment has revealed that the group delay ripple (GDR) is also reduced. 
For instance, the group delay ripple is caused by weak reflection occurring at the edges and along the grating, and this turns to be a critical parameter for compensation in a communication link. For the excitation at $f=117 \mathrm{kHz}$, it has been observed that for loads varying from $6 \mathrm{~V}$ to $10 \mathrm{~V}$ the GDR coefficient varies from $\pm 10.06 \mathrm{ps}$ (at $6 \mathrm{~V}$ ) to $\pm 5.55 \mathrm{ps}$ (at $10 \mathrm{~V}$ ), which is within or below that of many commercially specified compensators $(\sim \pm 10 \mathrm{ps})$.

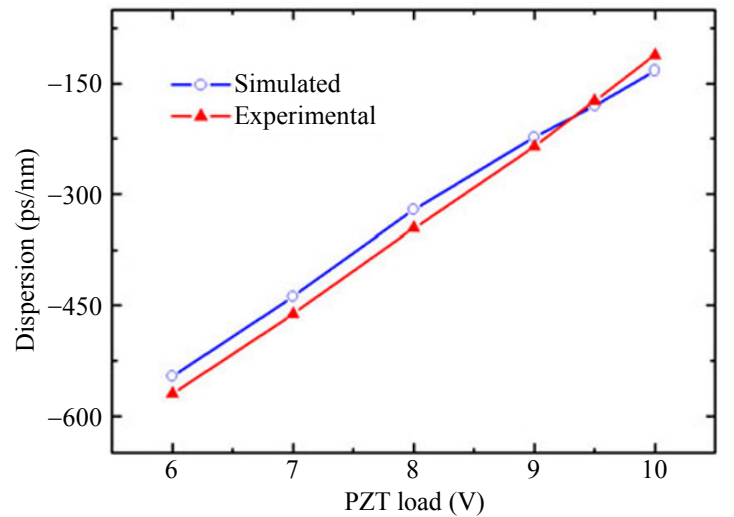

Fig. 17 Simulation and experimental dispersion behaviors as a function of the PZT load, when a flexural wave excites the fiber at $f=117 \mathrm{kHz}[18]$.

In summary, by adjusting the frequency and applied voltage, one turns the acousto-optic modulator into a low-power, fast device for the dynamic control of the group delay and the dispersion parameter.

\subsection{Writing of gratings with acoustic waves}

The writing of fiber gratings with complex profiles has driven the attention of several researchers as they are specially praised in optical signal processing and wavelength division multiplexing (WDM) optical communication systems. Several techniques were developed to fabricate such gratings, and one of the most used is the method that modulates the light intensity of the ultraviolet (UV) beam as it is scanned over the phase-mask [38, 71]. However, flexural and longitudinal waves can also be used to control the writing process of gratings in fibers offering new degrees of control as compared to well-established methods. When acoustic waves propagate along optical fibers, different strain profiles are achieved depending on the nature of the mode (longitudinal or flexural), and different effects on gratings inscribed in fibers are observed. For instance, a longitudinal wave interacting with an FBG creates compression and rarefaction zones along the fiber axis and modifies the grating planes. The effect causes additional bands to appear on both sides of the grating reflection spectrum as a result of the sinusoidal sampling modulation of both average refractive index and the grating pitch $[18,40]$. On the other hand, if flexural waves at low frequency modulate the fiber, the group delay and the bandwidth of the FBG spectral response will be changed. However, all these effects disappear as soon as the acoustic excitation is turned off.

However, with the insertion of the acousto-optic modulator in the writing setup (as shown in Fig. 18) and with the control of the acoustic wave propagation during the grating inscription process, one is able to achieve permanent non-periodic structures in the guide. Thus, the induced refractive index change generated by the conventional UV illuminating process in the grating inscription is also altered by the corresponding stress profile produced by the acoustic field, so that controlled sampled gratings are fabricated [72]. Figure 19 illustrates the effects obtained in the grating spectra using such a technique under the excitation of a longitudinal wave. The figure shows the resultant FBG spectrum when the acoustic wave is switched-off after the writing process for $1.021 \mathrm{MHz}$ (solid line) and $919 \mathrm{kHz}$ (dashed line) excitation frequencies, respectively. The case when no acoustic wave excites the fiber during the writing process is also shown in Fig. 19 by the dotted line. The created index modulation and grating pitch produce a non-uniform period that depends on the acoustic excitation period $\Lambda_{a}$. 


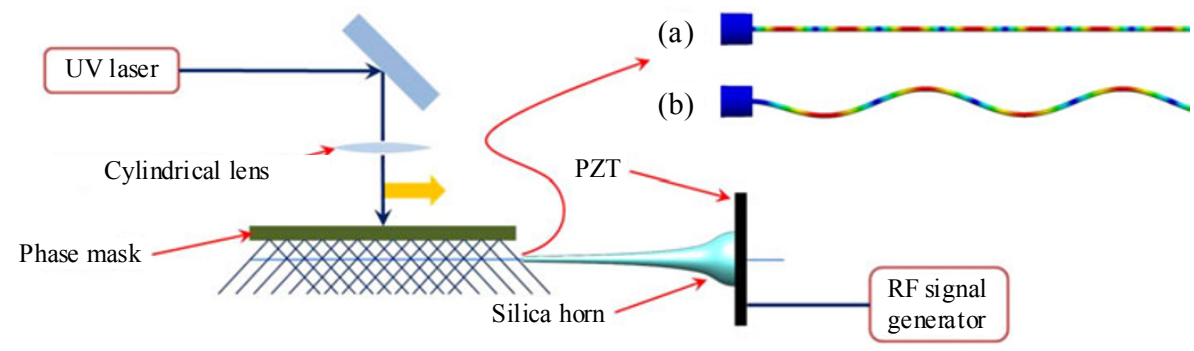

Fig. 18 Illustration of the acousto-optic modulator positioned in the grating writing setup showing the fiber under the phase-mask $[18]$.

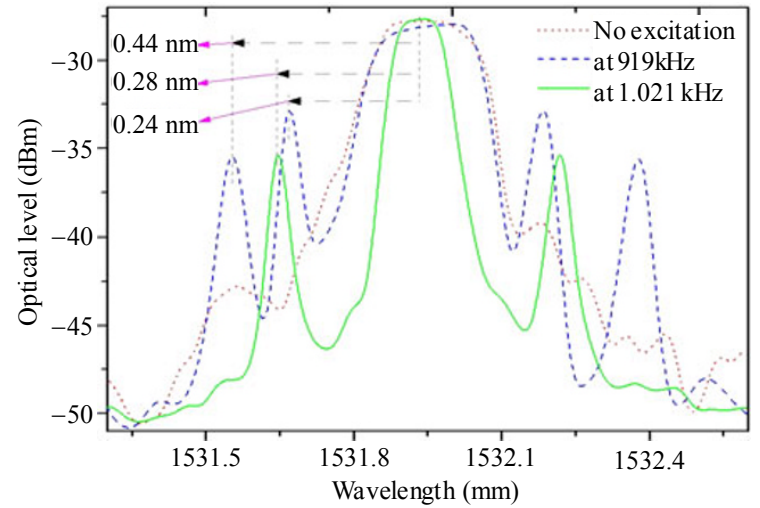

Fig. 19 FBG spectrum for the case when no acoustic wave excites the fiber during the writing process (dotted line) and when the longitudinal wave excites the fiber at $919 \mathrm{kHz}$ (dashed line) and $1.021 \mathrm{MHz}$ (solid line) and creates the side lobes, after the inscription is finished [72].

Moreover, when a flexural wave excites the fiber during the grating inscription, a periodic bending is generated [11]. The bending causes the misalignment of the UV interference pattern (generated by the inscription laser beam) in certain regions of the fiber core, so that no grating is formed. When the fiber is at rest, the UV interferogram produces the usual modulation of the refractive index in the fiber core. However, when the fiber is excited with a flexural mode [Fig. 18(b)], the core is dithered, thus destroying the UV fringe pattern consequently, no grating is inscribed. This effect can be used to create sampled gratings, narrow fiber cavities and phase-shifted gratings [73, 74]. The first sampled fiber gratings were written by sampling the period using a hair comb [75] and shortly after by turning on and off a scanning laser beam either with a fixed amplitude mask or by modulating the laser beam during scanning [76]. To achieve the same result by acoustic modulation, as the UV writing beam is scanned over the phase mask, the flexural wave is periodically turned on and off using the burst function of the signal generator.

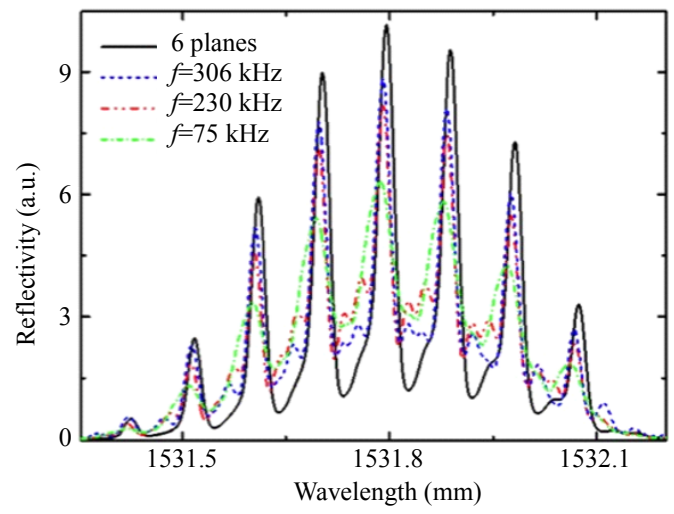

Fig. 20 Sampled grating written using a direct beam modulation technique (solid curve) and by means of flexural acoustic waves [73].

Figure 20 shows the resultant reflection spectra for different excitation frequencies (when flexural modes are excited) applied to the modulator. Initially, for comparison purposes, six gratings were inscribed using the conventional technique of modulating the UV writing beam (no acoustic wave excited the fiber) as it was scanned across the phase mask - the combined spectra are shown in the solid curve of Fig. 20. Employing now the acoustic technique, the length and number of gratings can be set by controlling the acoustic frequency. For example, considering $f=306 \mathrm{kHz}$, approximately four gratings are written in a 5-cm-length piece of fiber, 
whereas for $f=75 \mathrm{kHz}$, two gratings are obtained. If the frequency is kept constant and the burst time is tuned, this leads to a change in the number of gratings generated and also a change in the length of each grating. This methodology is also used to create phase-shifted gratings [74, 77].

The permanently imprinted sampled gratings are useful for creating complex filter devices suitable for signal multiplexing and demultiplexing or for serving as multiple narrowband transmission filters in photonic applications [78].

\subsection{Fiber viscometer}

Viscosity is the property of materials characterized by their resistance to flow. It is the property used to predict a number of other physical, chemical and biological parameters such as density, chemical content, molecular weight and color of materials. Viscometers are instruments used to measure viscosity and are particularly employed in the food and pharmaceutical industries to monitor and control processes during the fabrication of the food and drugs. The most common viscometers used in industries are based on electro-mechanical techniques having the rotation and vibration of a probe inside the fluid as the operating principle [79, 80]. An interesting and potential commercial alternative to this conventional approach is offered by an optical viscometer based on the acousto-optic effect [81].

Along with an LPG, an acousto-optic modulator can be used as a sensing element. For instance, long period gratings are able to measure changes in the external refractive index, which are manifested through a change in the grating dip wavelength and minimum transmissivity. Although viscosity can be related to and measured by the induced wavelength $(\Delta \lambda)$ and transmittance $(\Delta T)$ shift imparted to the LPG as a function of the concentration, the complex resulting behavior turns these parameters impractical for sensing. However, the measurement of viscosity can also be obtained using the grating temporal response. This is achieved by using the acousto-optic modulator to excite a flexural acoustic wave, forming bends along the fiber and the grating. As described in Section 4.3, the effect causes the shifting in the LPG rejection band and changes the maximum band transmissivity. The measurement of viscosity is obtained by observing the optical response that critically depends on the damping of the acoustic wave by the surrounding medium. The damped response to the applied signal is a direct measure of the relaxation time associated with viscous flow - the more viscous the flow the slower the relaxation after the impulse signal is applied to the grating. The schematic diagram in Fig. 21 shows how the viscosity damps the acoustic wave. Figure 21(a) shows the silica-horn fiber at rest, and Fig. 21(b) shows the formation of a flexural wave. The following figures show how the acoustic wave damps, considering an increasing in the viscosity $(\eta)$ of the environment where the fiber is immersed.

(a)

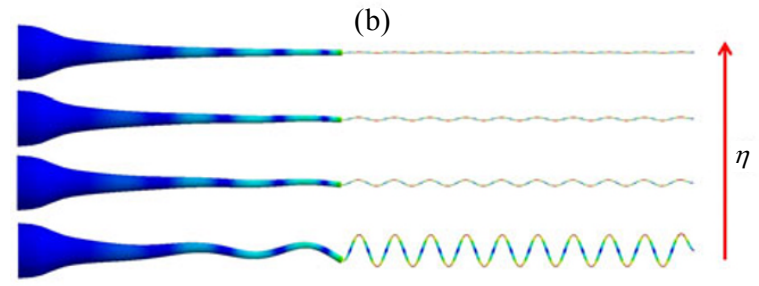

Fig. 21 Illustration of the acoustic behavior of the silica-horn /fiber set when (a) at rest and (b) acousticly excited, considering an increase in the external viscosity [18].

For testing the viscometer principle, the measurement has been performed in anhydrous $\mathrm{D}(+)$-glucose $\left(\mathrm{C}_{6} \mathrm{H}_{12} \mathrm{O}_{6}\right)$ in de-ionised, distilled water. This is a solution with a well-known refractive index and viscosity as a function of the concentration and is often used to characterize and calibrate commercial devices [82].

The measurement requires the switching of the acoustic flexural wave in the solution using the burst 
mode of a signal generator. The idea is to probe the LPG response within a short interaction time. When this happens, the LPG spectrum is shifted, and a maximum signal level in the photodetector is quickly reached. The analysis is based on observing the grating temporal response. Figure 22 shows the dynamic behavior of the LPG when immersed in the solution with $\left[\mathrm{C}_{6} \mathrm{H}_{12} \mathrm{O}_{6}\right]=4023 \mathrm{~mol} / \mathrm{L}$ for 100 cycles of acoustic excitation at $f=39.9 \mathrm{kHz}$. As seen from the figure, the transition time, $\tau_{r}$, corresponds to the first excitation of the fiber. However, a relaxation of the fiber, corresponding to the damping by the viscous solution is expected, which changes the spectral shift. This corresponds to a small signal reduction in the grating response. But because this measurement is time dependent, there is a finite time, $\tau_{s}^{\prime}$, before the solution viscosity resists and subsequently relaxes the fiber until there is a full relaxation at $\tau_{s}$, after which a standing wave is achieved. Then, a method is necessary to separate out the excitation and relaxation time by only measuring $\tau_{r}$ or $\tau_{s}^{\prime}=\left(\tau_{s}-\tau_{r}\right)$ in order to obtain a linear relationship between the solution concentration and the time and, therefore, $\eta$. The time at which the photodetector signal remains constant is labeled as $\tau_{c}$ and is related to the inertia of the fiber after the solution has relaxed around it and corresponds to the generation of a standing wave. When the acoustic wave is finally switched off, the LPG relaxes and experiences the fall time, $\tau_{f}$, back to the rest state.

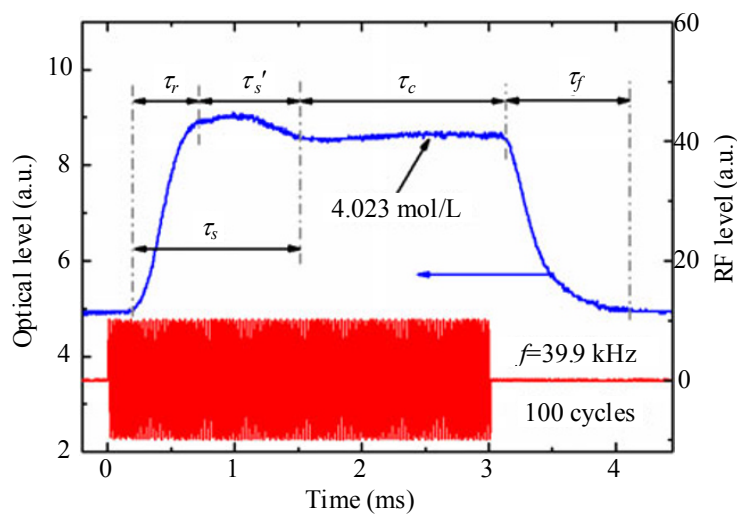

Fig. 22 Time parameters considering a solution with $\left[\mathrm{C}_{6} \mathrm{H}_{12} \mathrm{O}_{6}\right]=4023 \mathrm{~mol} / \mathrm{L}[81]$.
Figure 23 shows the behavior of the viscosity and rise time depending on the glucose concentration. One can see that the viscosity is directly related to $\left[\mathrm{C}_{6} \mathrm{H}_{12} \mathrm{O}_{6}\right]$ through a quadratic dependence (LIDE, 2008). Given that both the rise time and concentration have a quadratic dependence, this means that the viscosity has a linear dependence upon the rise time. Figure 24 shows the linear dependence for both the rise time and the total relaxation time, $\tau_{s}$, upon the viscosity. It is observed that both increases, when $\eta$ increases, are consistent with the damping of the acoustic wave by the solution as expected ideally. These linear relations therefore make viscosity measurement straightforward, offering a fully new way of sensing this parameter.

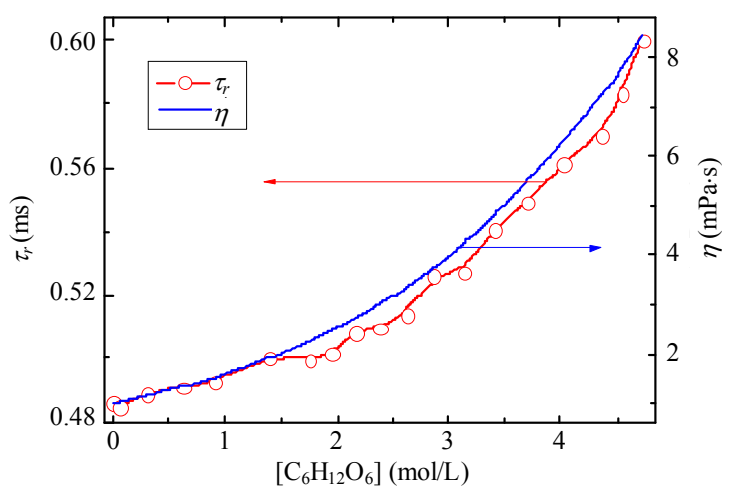

Fig. 23 Quadratic relationship between the glucose concentration and rise time and viscosity [81].

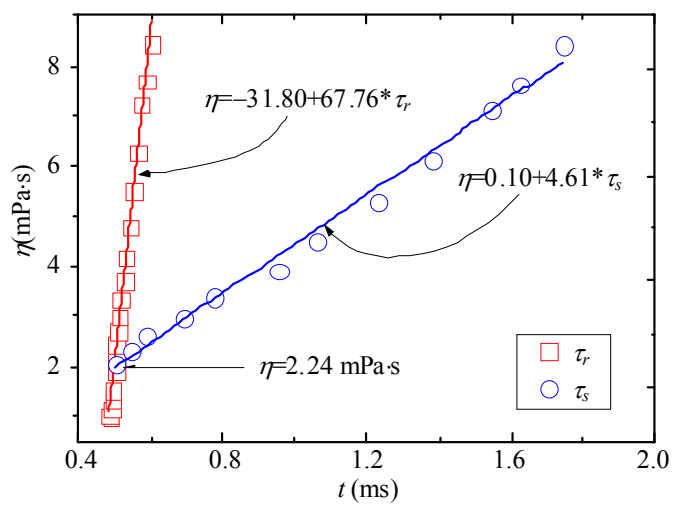

Fig. 24 Viscosity versus time behavior [81].

\section{Conclusions}

The adequate use of the acousto-optic effect 
depends on the excitation of a particular acoustic mode, which is achieved by providing the correct frequency and amplitude to the acousto-optic modulator. Previously, however, the characterization of the resonances of the piezoelectric element is useful in order to identify the relevant resonant modes that are further employed in the acoustic excitation. By using a numerical approach based on the finite elements and the transfer matrix methods, one is able to model the acousto-optic modulator and simulate the effect. Flexural and longitudinal modes can be excited using a configuration, where the fiber and the modulator are axially aligned. The interaction of the propagating acoustic mode with Bragg and long period gratings inscribed in the fiber extends the range of applications, which has led to the development of tunable filters, dynamic gain equalizers, the control of laser parameters and new fiber sensors.

For instance, in the low frequency regime $(<250 \mathrm{kHz})$, when flexural modes dominate, the interaction of such a mode with an FBG causes the chirp of the grating spectrum. A decay of the grating reflectivity is also observed in some cases, revealing a coupling of the optical guided mode to radiated modes in the cladding. The interaction of flexural modes with LPGs makes it possible to induce a shift in the rejection band to longer wavelengths and to change its transmissivity by controlling the intensity of the applied acoustic field.

On the other hand, when longitudinal modes are excited, the period of the compression and rarefaction strain field in the fiber becomes shorter. Such a field will not only cause an additional change in the effective indices of the optical forward and backward propagating modes of a Bragg grating, but also modulate its pitch, leading to the appearance of additional bands on both sides of the grating reflection spectrum. Simulation results obtained using the FEM and TMM techniques show a very good agreement with experimental observations.
In recent years, new applications of the acousto-optic mechanism have been developed, such as the technique for writing gratings under the acousto-optic excitation and a new type of viscometer. Several other devices and applications have been also proposed and demonstrated, such as the application of the mechanism in the control of the Q-switching [59] and mode-locking [62] in laser cavities and its use for dispersion compensation in optical communication links. Although the mechanism is well-known, the study and design of acousto-optic modulators is still a topic that deserves attention, particularly if one wishes to achieve high reproducibility. The experimental characterization of acoustic modes is also an area that requires further investigation, as the excitation of a specific mode impacts the way optical devices operate.

\section{Acknowledgment}

The authors would like to thank $\mathrm{CNPq}$ and CAPES, Brazil, the Fundação para a Ciência e Tecnologia (FCT), Portugal, and the Australian Research Council (ARC) for supporting this work. The authors also would like to thank Prof. Marcos Antonio Ruggieri Franco from IEAv/CTA, Brazil, for helpful discussions on the simulation of the acousto-optic modulator, Prof. Jucélio Tomás Pereira and Prof. Carlos Alberto Bavastri, both from the Federal University of Paraná, Brazil, for the initial discussions concerning the propagation and simulation of acoustic waves in thin rods.

Open Access This article is distributed under the terms of the Creative Commons Attribution License which permits any use, distribution, and reproduction in any medium, provided the original author(s) and source are credited.

\section{References}

[1] A. Yariv and P. Yeh, Optical waves in crystals. New 
York: John Wiley \& Sons, Inc., 1984.

[2] L. Pochhammer, "Über die Fortpflanzungsgeschwindigkeiten kleiner Schwingungen in einem unbegrenzten isotropen Kreiscylinder," Journal für reine und angewandt Math. (Crelle), vol. 81, pp. 324-336, 1876.

[3] C. Chree, "The equations on an isotropic elastic solid in polar and cylindrical coordinates, their solutions, and applications," Transactions of the Cambridge Philosophical Society, vol. 14, pp. 250-369, 1889.

[4] J. D. Achenbach, Wave Propagation in Elastic Solids. Amsterdam: North-Holland Publishing Company, 1973.

[5] L. Meirovitch, Elements of vibration analysis. Singapore: The McGraw-Hill Company, 1986.

[6] J. S. Rao, Advanced theory of vibration. New Delhi, India: John Wiley \& Sons, Inc., 1992.

[7] R. N. Thurston, "Elastic waves in rods and clad rods," Journal of the Acoustic Society of America, vol. 64, no. 1, pp. 1-37, 1978.

[8] S. A. Zemon and M. L. Dakss, "Acoustoptic modulator for optical fiber waveguides," U. S. Patent 4068191, 1978.

[9] H. Jaffe, "Piezoelectric ceramics," Journal of the American Ceramic Society, vol. 41, no. 11, pp. 494-498, 1958.

[10] H. Jaffe and D. A. Berlincourt, "Piezoelectric transducer materials," Proceedings of the IEEE, vol. 53, no. 10, pp. 1372-1386, 1965.

[11] R. A. Oliveira, P. T. Neves, J. T. Pereira, J. Canning, and A. A. P. Pohl, "Vibration mode analysis of a silica horn fiber Bragg grating device," Optics Communications, vol. 283, no. 7, pp. 1296-1302, 2010.

[12] M. Berwick, C. N. Pannell, P. St. J. Russell, and D. A. Jackson, "Demonstration of birefringent optical fibre frequency shifter employing torsional acoustic waves," Electronics Letters, vol. 27, no. 9, pp. 713-715, 1991.

[13] K. J. Lee, K. S. Hong, H. C. Park, and B. Y. Kim, "Polarization coupling in a highly birefringent photonic crystal fiber by torsional acoustic wave," Optics Express, vol. 16, no. 7, pp. 4631-4638, 2008.

[14] H. E. Engan, B. Y. Kim, J. N. Blake, and H. J. Shaw, "Optical frequency shifting in two-mode optical fibers by flexural acoustic waves," IEEE Ultrasonic Symposium, pp. 435-438, 1986.

[15] W. F. Liu, P. St. J. Russell, and L. Dong, "Acousto-optic superlattice modulator using a fiber Bragg grating," Optics Letters, vol. 22, no. 19, pp. 1515-1517, 1997.
[16] H. E. Engan, B. Y. Kim, J. N. Blake, and H. J. Shaw, "Propagation and optical interaction of guided acoustic waves in two-mode optical fibers," Journal of Lightwave Technology, vol. 6, no. 3, pp. 428-436, 1988.

[17] J. N. Blake, B. Y. Kim, H. E. Engan, and H. J. Shaw, "Analysis of intermodal coupling in a two-mode fiber with periodic microbends," Optics Letters, vol. 12, no. 4, pp. 281-283, 1987.

[18] R. A. Oliveira, "Characterization and new applications of the acousto-optic effect in fiber gratings," Ph.D. dissertation, Federal University of Technology - Paraná, 2011.

[19] A. H. Meitzler, H. M. Jr. O'Bryan, and H. F. Tiersten, "Definition and measurement of radial mode coupling factors in piezoelectric ceramic materials with large variations in Poisson's Ratio," IEEE Transactions on Ultrasonics Ferroelectrics and Frequency Control, vol. 20, no. 3, pp. 233-239, 1973.

[20] M. Brissaud, "Characterization of piezoceramics," IEEE Transactions on Ultrasonics Ferroelectrics and Frequency Control, vol. 38, no. 6, pp. 603-617, 1991.

[21] M. Brissaud, "Three-dimensional modeling of piezoelectric materials," IEEE Transactions on Ultrasonics Ferroelectrics and Frequency Control, vol. 57, no. 9, pp. 2051-2065, 2010.

[22] X. H. Du, Q. M. Wang, and K. Uchino, “An accurate method for the determination of complex coefficients of single crystal piezoelectric resonators II: design of measurement and experiments," IEEE Transactions on Ultrasonics Ferroelectrics and Frequency Control, vol. 51, no. 2, pp. 238-248, 2004.

[23] A. Baliato, "Modeling piezoelectric and piezomagnetic devices and structures via equivalent networks," IEEE Transactions on Ultrasonics Ferroelectrics and Frequency Control, vol. 48, no. 5, pp. 1189-1240, 2001.

[24] Available from: http://www.comsol.com.

[25] Available from:http://www.ansys.com.

[26] Y. Jiang, "High-resolution interrogation technique for fiber optic extrinsic Fabry-Perot interferometric sensors by the peak-to-peak method," Applied Optics, vol. 47, no. 7, pp. 925-932, 2008.

[27] Y. Huang, T. Wei, Z. Zhou, Y. Zhang, G. Chen, and H. Xiao, "An extrinsic Fabry-Perot interferometer-based large strain sensor with high resolution," Measurement Science and Technology, vol. 21, no. 10, pp. 105308, 2010.

[28] R. E. Silva and A. A. P. Pohl, "Characterization of 
flexural acoustic waves in optical fibers using an extrinsic Fabry-Perot interferometer," Measurement Science and Technology, vol. 23, no. 5, pp. 055206, 2012.

[29] R. E. Silva and A. A. P. Pohl, "Characterization of longitudinal acoustic waves in a fiber using an extrinsic Fabry-Perot interferometer," presented at the 22nd International Conference on Optical Fiber Sensors (OFS), Beijing, China, Oct. 15-19, 2012.

[30] A. W. Snyder and J. D. Love, Optical Waveguide Theory. New York, USA: Chapman and Hall Ltd., pp. $542,1983$.

[31] H. F. Taylor, "Bending effects in optical fibers," Journal of Lightwave Technology, vol. LT-2, no. 5, pp. 617-627, 1984.

[32] B. Y. Kim, J. N. Blake, H. E. Engan, and H. J. Shaw, "All-fiber acousto-optic frequency shifter," Optics Letters, vol. 11, no. 6, pp. 389-391, 1986.

[33] T. A. Birks, P. St. J. Russel, and D. O. Culverhouse, "The acousto-optic effect in single-mode fiber tapers and couplers," Journal of Lightwave Technology, vol. 14, no. 11, pp. 2519-2529, 1996.

[34] T. Matsui, K. Nakajima, K. Shiraki, and T. Kurashima, "Ultra-broadband mode conversion with acousto-optic coupling in hole-assisted fiber," Journal Lightwave Technology, vol. 27, no. 13, pp. 2183-2188, 2009.

[35] T. A. Birks, P. St. J. Russell, and C. N. Pannell, "Low power acousto-optic device based on a tapered single mode fiber," IEEE Photonics Technology Letters, vol. 6, no. 6, pp. 725-727, 1994.

[36] R. Feced, C. Alegria, M. N. Zervas, and R. I. Laming, "Acousto-optic attenuation filters based on tapered optical fibers," IEEE Journal Selected Topics in Quantum Electronics, vol. 5, no. 5, p. 1278-1288, 1999.

[37] J. Zhao and X. Liu, "Fiber acousto-optic mode coupling between the higher-order modes with adjacent azimuthal numbers," Optics Letters, vol. 31, no. 11, pp. 1609-1611, 2006.

[38] A. Othonos and K. Kalli, Fiber Bragg gratings fundamentals and applications in telecommunications and sensing. Boston: Artech House, 1999.

[39] T. Erdogan, "Fiber grating spectra," Journal of Lightwave Technology, vol. 15, no. 8, pp. 1277-1294, 1997.

[40] W. F. Liu, P. St. J. Russell, and L. Dong, "Acousto-optic superlattice modulator using a fiber Bragg grating," Optics Letters, vol. 22, no. 19, pp. 1515-1517, 1997.
[41] P. St. J. Russell and W. F. Liu, "Acousto-optic superlattice modulation in fiber Bragg gratings," Journal Optics Society of America A, vol. 17, no. 8, pp. 1421-1429, 2000.

[42] O. C. Zienkiewicz and R. L. Taylor, The finite element method, Volume 1: The basis. Oxford: Butterworth-Heinemann, 2000.

[43] M. Yamada and K. Sakoda, "Analysis of almost-periodic distributed feedback slab waveguides via a fundamental matrix approach," Applied Optics, vol. 26, no. 16, pp. 3474-3478, 1987.

[44] F. Abrishamian, S. Sato, and M. Imai, "A new method of solving multimode coupled equations for analysis of uniform and non-uniform fiber Bragg gratings and its application to acousticly induced superstructure modulation," Optical Review, vol. 12, no. 6, pp. 467-471, 2005.

[45] R. A. Oliveira, P. T. NevesJr, J. T. Pereira, and A. P. P. Pohl, "Numerical approach for designing a Bragg grating acousto-optics modulator using finite element and transfer matrix methods," Optics Communications, vol. 281, no. 19, pp. 4899-4905, 2008.

[46] A. A. P. Pohl, K. Cook, and J. Canning, "Acoustic-induced modulation of photonic crystal fibre Bragg gratings," In Proceedings of the 10th International Conference on Transparent Optical Networks, Athen, Greece, vol. 2, pp. 51-54, 2008.

[47] M. Vengsarkar, P. J. Lemaire, J. B. Judkins, V. Bhatia, T. Erdogan, and J. E. Sipe, "Long-period fiber gratings as band-rejection filter," Journal Lightwave Technology, vol. 14, no. 1, pp. 58-65, 1996.

[48] S. W. James and R. P. Tatam, "Optical fibre long-period grating sensors: characteristics and application," Measurement Science and Technology, vol. 14, no. 5, pp. R49-R61, 2003.

[49] G. Rego, P. Marques, J. Santos, and H. Salgado, "Arc-induced long-period grating," Fiber and Integrated Optics, vol. 24, no. 3-4, pp. 245-259, 2005.

[50] R. C. Kamikawachi, G. R. Possetti, R. Falate, M. Muller, and J. L. Fabris, "Influence of surrounding media refractive index on the thermal and strain sensitivities of long period gratings," Applied Optics, vol. 46, no. 35, pp. 2831-2837, 2007.

[51] C. F. Beards, Structural vibration: analysis and damping, 1st edition, Butterwoth-Heinemann, 1996.

[52] R. A. Oliveira, G. R. C. Posseti, C. A. F. Marques, P. T. Never, K. Cook, R. C. Kamikawachi, et al., "Control of the long period grating spectrum through 
low frequency flexural acoustic waves," Measurement Science \& Technology, vol. 22, no. 4, pp. 045205, 2011.

[53] H. S. Kim, S. H. Yun, H. K. Kim, N. Park, and B.Y. Kim, "Dynamic erbium-doped fiber amplifier based on active gain-flattening with fiber acousto-optic tunable filters," IEEE Photonics Technology Letters, vol. 11, no. 10, pp. 1229-1231, 1999.

[54] D. W. Huang, W. F. Liu, C. W. Wu, and C. C. Yang, "Reflectivity-tunable fiber Bragg grating reflectors," IEEE Photonics Technology Letters, vol. 12, no. 2, pp. 176-178, 2000.

[55] W. F. Liu, I. M. Liu, L. W. Chung, D. W. Huang, and C. C. Yang, "Acoustic-induced switching of the reflection wavelength in a fiber Bragg grating," Optics Letters, vol. 25, no. 18, pp. 1319-1321, 2000.

[56] W. F. Liu and P. J. Tu, "Switchable narrow-bandwidth comb filters based on an acousto-optic superlattice modulator in sinc-sampled fiber gratings," Optical Engineering, vol. 40, no. 8, pp. 1513-1515, 2001.

[57] A. Diez, M. Delgado-Pinar, J. Mora, J. L. Cruz, and M. V. Andrés, "Dynamic fiber-optic add-drop multiplexer using Bragg gratings and acousto-optic-induced coupling," IEEE Photonics Technology Letters, vol. 15, no. 1, pp. 84-86, 2003.

[58] D. I. Yeom, H. S. Park, and B. Y. Kim, "Tunable narrow-bandwidth optical filter based on acousticly modulated fiber Bragg grating," IEEE Photonics Technology Letters, vol. 16, no. 5, pp. 1313-1315, 2004.

[59] M. Delgado-Pinar, D. Zalvidea, A. Díez, P. Pérez-Millán, and M. V. Andrés, "Q-switching of an all-fiber laser by acousto-optic modulation of a fiber Bragg grating," Optics Express, vol. 14, no. 3, pp. 1106-1112, 2006.

[60] Z. Luo, C. Ye, Z. Cai, X. Dai, Y. Kang, and H. Xu, "Numerical analysis and optimization of optical spectral characteristics of fiber Bragg gratings modulated by a transverse acoustic wave," Applied Optics, vol. 46, no. 28, pp. 6959-6965, 2007.

[61] C. A. F. Marques, R. A. Oliveira, A. P. P. Pohl, J. Canning, and R. N. Nogueira, "Dynamic control of a phase-shifted FBG through acousto-optic modulation," Optics Communications, vol. 284, no. 5, pp. 1228-1231, 2011.

[62] C. Cuadrado-Laborde, A. Diez, M. Delgado-Pinar, J. L. Cruz, and M. V. Andrés, "Mode-Locking of an all-fiber laser by acousto-optic superlattice modulation," Optics Letters, vol. 34, no. 7, pp.
1111-1113, 2009.

[63] L. Grüner-Nielsen, S. N. Knudsen, B. Edvold, T. Veng, D. Magnussen, C. C.Larsen, et al., "Dispersion compensating fibers," Optical Fiber Technology, vol. 6, no. 2, pp. 164-180, 2000.

[64] Y. Painchaud, C. Paquet, and M. Guy, "Optical tunable dispersion compensators," Optics and Photonics News, vol. 18, no. 9, pp 48-53, 2007.

[65] N. M. Litchinitser, B. J. Eggleton, and D. V. Patterson, "Fiber Bragg gratings for dispersion compensation in transmission: theoretical model and design criteria for nearly ideal pulse recompression," Journal of Lightwave Technology, vol. 15, no. 8, pp. 1303-1319, 1997.

[66] J. F. Brennan III, "Broadband fiber Bragg gratings for dispersion management," Journal of Optical and Fiber Communications Report, vol. 2, no. 5, pp. 397-434, 2005.

[67] M. Sumetsky and B. J. Eggleton, "Fiber Bragg gratings for dispersion compensation in optical communication systems," in Ultra-High Speed Optical Transmission Technology, Springer Science + Business Media Inc., vol. 3, pp. 277-299, 2007.

[68] J. Lauzon, S. Thibault, J. Martin, and F. Oullette, "Implementation and characterization of fiber Bragg gratings linearly chirped by a temperature gradient," Optics Letters, vol. 19, no. 23, pp. 2027-2029, 1994.

[69] B. J. Eggleton, A. Ahuja, P. S. Westbrook, J. A. Rogers, P. Kuo, T. N. Nielsen, et al., "Integrated tunable fiber gratings for dispersion management in high-bit rate systems," Journal of Lightwave Technology, vol. 18, no. 10, pp. 1418-1432, 2000.

[70] Y. J. Lee, J. Bae, K. Lee, Je-M. Jeong, and S. B. Lee, "Tunable dispersion and dispersion slope compensator using strain-chirped fiber Bragg grating," IEEE Photonics Technology Letters, vol. 19, no. 10, pp. 762-764, 2007.

[71] K. O. Hill and G. Meltz, "Fiber Bragg grating technology fundamentals and overview," Journal of Lightwave Technology, vol. 15, no. 8, pp. 1263-1276, 1997.

[72] R. A. Oliveira, K. Cook, J. Canning, and A. A. P. Pohl, "Bragg grating writing in acoustically excited optical fiber," Applied Physics Letters, vol. 97, no. 4, pp. 041101, 2010.

[73] R. A. Oliveira, K. Cook, C. A. F. Marques, J. Canning, R. N. Nogueira, and A. A. P. Pohl, "Complex Bragg grating writing using direct modulation of the optical fibre with flexural acoustic 
waves," Applied Physics Letters, vol. 99, no. 16, pp. 161111, 2011.

[74] J. Canning, H. J. Deyerl, and M. Kristensen, "Precision phase-shifting applied to fiber Bragg gratings," Optics Communications, vol. 244, no. 1-6, pp. 187-191, 2005.

[75] P. Hill, J. Canning, B. Eggleton, M. G. Sceats, private communication.

[76] B. J. Eggleton, P. A. Krug, L. Poladian, and F. Ouellete, "Long periodic superstructures Bragg gratings in optical fibres," Electronics Letters, vol. 30, no. 19, pp. 1620-1622, 1994.

[77] J. Canning and M. G. Sceats, " $\pi$-phase-shifted periodic distributed structures in optical fibers by UV postprocessing," Electronics Letters, vol. 30, no. 16, pp 1344-1345, 1994.

[78] H. Li, M. Li, Y. Sheng, and J. E. Rothemberg, "Advances in the design and fabrication of high-channel count fiber Bragg gratings," Journal of Lightwave Technology, vol. 25, no. 9, pp. 2739-2750, 2007.

[79] E. M. Barber, J. R. Muenger, and F. J. Villforth, "High rate of shear rotational viscometer," Analytical Chemistry, vol. 27, no. 3, pp. 425-429, 1955.

[80] O. Susuki, S. Ishiwata, M. Hayashi, and H. Oshima, "Vibration type rheometer apparatus," U. S. Patent 4941346, 1990.

[81] R. A. Oliveira, J Canning, K. Cook, M. Naqshbandi, and A. A. P. Pohl, "Compact dip-style viscometer based on the acousto-optic effect in a long period fibre grating," Sensors and Actuators. B: Chemical, vol. 157, no. 2, pp. 621-626, 2011.

[82] D. R. Lide, CRC handbook of chemistry and physics, 89th edition. CRC Press, Boca raton, Fl, USA, 2008. 